\title{
A Framework for the Cryptographic Verification of Java-like Programs
}

\section{Technical Report}

\author{
Ralf Küsters \\ University of Trier, Germany \\ kuesters@uni-trier.de
}

\author{
Tomasz Truderung \\ University of Trier, Germany \\ truderung@uni-trier.de
}

\author{
Jürgen Graf \\ KIT, Germany \\ graf@kit.edu
}

\begin{abstract}
We consider the problem of establishing cryptographic guarantees-in particular, computational indistinguishability - for Java or Java-like programs that use cryptography. For this purpose, we propose a general framework that enables existing program analysis tools that can check (standard) non-interference properties of Java programs to establish cryptographic security guarantees, even if the tools a priori cannot deal with cryptography. The approach that we take is new and combines techniques from program analysis and simulation-based security. Our framework is stated and proved for a Java-like language that comprises a rich fragment of Java. The general idea of our approach should, however, be applicable also to other practical programming languages.

As a proof of concept, we use an automatic program analysis tool for checking non-interference properties of Java programs, namely the tool Joana, in order to establish computational indistinguishability for a Java program that involves clients sending encrypted messages over a network, controlled by an active adversary, to a server.
\end{abstract}

\section{INTRODUCTION}

In this paper, we consider the problem of establishing security guarantees for Java or Java-like programs that use cryptography, such as encryption. More specifically, the security guarantees we are interested in are computational indistinguishability properties: Two systems $S_{1}$ and $S_{2}$ coded in Java, i.e., two collections of Java classes, are computationally indistinguishable if no probabilistic polynomially bounded environment (which is also coded as a Java program) is able to distinguish, with more than negligible probability, whether it interacts with $S_{1}$ or $S_{2}$. As a special case, $S_{1}$ and $S_{2}$ might only differ in certain values for certain variables. In this case, the computational indistinguishability of $S_{1}$ and $S_{2}$ means that the values of these variables are kept private, a property referred to as privacy, anonymity, or strong secrecy. Indistinguishability is a fundamental security property relevant in many security critical applications, such as secure message transmission, key exchange, anonymous communication, e-voting, etc.

Our goal. The main goal of this paper is to develop a general framework that allows us to establish cryptographic indistinguishability properties for Java programs using existing program analysis tools for analyzing (standard) non- interference properties [17] of Java programs, such as the tools Joana [20], KeY [1], a tool based on Maude [3], and Jif [29], [30]. As such, our work also contributes to the problem of implementation-level analysis of crypto-based software (such as cryptographic protocols) that has recently gained much attention (see Sections $\mathrm{X}$ and $\mathrm{XI}$ ).

A fundamental problem that we face is that existing program analysis tools for non-interference properties cannot deal with cryptography directly. In particular, they typically do not deal with probabilities and the non-interference properties that they prove are w.r.t. unbounded adversaries, rather than probabilistic polynomially bounded adversaries. For example, if a message is encrypted and the ciphertext is given to the adversary, the tools consider this to be an illegal information flow (or a declassification), because a computationally unbounded adversary could decrypt the message. This problem has long been observed in the literature (see, e.g., [36] and references therein).

Our approach. Our approach to enabling these tools to nevertheless deal with cryptography and in the end provide cryptographic security guarantees is to use techniques from simulation-based security (see, e.g., [10], [35], [24]). The idea is to first analyze a (deterministic) Java program where cryptographic operations (such as encryption) are performed within ideal functionalities. Such functionalities typically provide guarantees even in the face of unbounded adversaries and can often be formulated without probabilistic operations. As we show as part of our framework, we can then replace the ideal functionalities by their realizations, obtaining the actual Java program (without idealized components) with cryptographic guarantees.

Our contribution in more detail. More precisely, our approach and the contribution of this paper are as follows.

Our framework is formulated for a language we call Jinja+ and is proven w.r.t. the formal semantics of this language. Jinja+ is a Java-like language that extends the language Jinja [23] and comprises a rich fragment of Java, including classes, inheritance, (static and non-static) fields and methods, the primitive types int, boolean, and byte (with the usual operators for these types), arrays, exceptions, 
and field/method access modifiers, such as public, private, and protected.

Along the lines of simulation-based security, we formulate, in Jinja+, rather than in a Turing machine model, what it means for two systems to be computationally indistinguishable, and for one system to realize another system. We then prove a composition theorem that allows us to replace ideal functionalities (formulated as Jinja+ systems) by their realizations (also formulated as Jinja+ systems) in a more complex system. The definitions and proofs need care because interfaces between different Jinja+ systems are classes with their fields and methods, and hence, these interfaces are very different and much richer than in the case of interactive Turing machines, where machines are simply connected by tapes on which bit strings are exchanged.

As mentioned before, we are mainly interested in establishing computational indistinguishability properties for crypto-based Java or Java-like programs (i.e., programs that use cryptography) using existing analysis tools for languagebased information flow analysis. At the core of our approach, sketched above, is a theorem that says that if two systems that both use an ideal functionality are perfectly indistinguishable, then these systems are computationally indistinguishable if the ideal functionality is replaced by its realization, where perfect indistinguishability is defined (for deterministic Java programs) just as computational indistinguishability but w.r.t. unbounded adversaries. Together with another theorem that we obtain, and which states that (termination-insensitive) non-interference [36] is equivalent to perfect indistinguishability, we obtain that by proving non-interference using existing program analysis tools, we can establish computational indistinguishability properties.

Many program analysis tools can deal only with closed Java programs. The systems we want to analyze are, however, open, because they interact with a network or use some libraries that we do not (have to) trust, and hence, do not have to analyze. In our setting, the network and such libraries are simply considered to be part of the environment (the adversary). As part of our framework, we therefore also propose proof techniques that help program analysis tools to deal with these kinds of open systems, and in particular, to prove non-interference properties about these systems. These techniques are used in our case study (see below), but they are rather general, and hence, relevant beyond our case study.

Since we use public-key encryption in our case study, we also propose an ideal functionality for public-key encryption coded in Jinja+, in the spirit of similar functionalities in the simulation-based approach (see, e.g., [10], [27]), and prove that it can be realized with any IND-CCA2-secure publickey encryption scheme. This result is needed whenever a Java system is analyzed that uses public-key encryption, and hence, is relevant beyond our case study. We note that the formulation of our ideal functionality is more restricted than the one in the cryptographic literature in that corruption is not handled within the functionality.

As a case study and as a proof of concept of our framework and approach, we consider a simple Java program, which in fact falls into the Jinja+-fragment of Java and in which clients (whose number is determined by an active adversary) encrypt secrets under the public key of a server and send them, over an untrusted network controlled by the active adversary, to a server who decrypts these messages. Using the program analysis tool Joana [20], which is a fully automated tool for proving non-interference properties of Java programs, we show that our system enjoys the non-interference property (with the secrets stored in high variables) when the ideal functionality is used instead of real encryption. The theorems proved in our framework thus imply that this system enjoys the computational indistinguishability property (in this case strong secrecy of the secrets) when the ideal functionality is replaced by its realization, i.e., the actual IND-CCA2-secure public-key encryption scheme.

Structure of the paper. The language Jinja+ is introduced in Section II. Perfect and computational indistinguishability for Jinja+ systems are formulated in Section III. In Section IV, we define simulation-based security for Jinja+ and present the mentioned composition theorem. The relationship between computational and perfect indistinguishability as well as non-interference is shown in Sections V and VI. The proof technique for non-interference in open systems is discussed Section VII. The ideal functionality for public-key encryption and its realization can be found in Section VIII, with the case study presented in Section IX. In Section X, we discuss related work. We conclude in Section XI. More details are provided in the appendix.

\section{JINJA+: A JAVA-LIKE LANGUAGE}

As mentioned in the introduction, our framework is stated for a Java-like language which we call Jinja+. Jinja+ is based on Jinja [23] and extends this language with some additional features that are useful or needed in the context of our framework.

Jinja+ covers a rich subset of Java, including classes, inheritance, (static and non-static) fields and methods, the primitive types int, boolean, and byte (with the usual operators for these types), arrays, exceptions, and field/method access modifiers, such as public, private, and protected. Among the features of Java that are not covered by Jinja+ are: abstract classes, interfaces, strings, and concurrency. We believe that extending our framework to work for these features of Java, except for concurrency, is quite straightforward. We leave such extensions for future work.

We now first recall the Jinja language and then present the extended language Jinja+. 


\section{A. Jinja}

Syntax. Expressions in Jinja are constructed recursively and include: (a) creation of a new object, (b) casting, (c) literal values (constants) of types boolean and int, (d) nutu, (e) binary operations, (f) variable access and variable assignment, (g) field access and field assignment, (h) method call, (i) blocks with locally declared variables, (j) sequential composition, (k) conditional expressions, (l) while loop, (m) exception throwing and catching.

A program or a system is a set of class declarations. A class declaration consists of the name of the class and the class itself. A class consists of the name of its direct superclass (optionally), a list of field declarations, and a list of method declarations, where we require that different fields and methods have different names. A field declaration consists of a type and a field name. A method declaration consists of the method name, the formal parameter names and types, the result type, and an expression (the method body). Note that there is no return statement, as a method body is an expression; the value of such an expression is returned by the method.

In what follows, by a program we will mean a complete program (one that is syntactically correct and can be executed). We assume that a program contains a unique static method main (declared in exactly one class); this method is the first to be called in a run. By a system we will mean a set of classes which is syntactically correct (can be compiled), but possibly incomplete (can use not defined classes). In particular, a system can be extended to a (complete) program.

Some constructs of Jinja (and the richer language Jinja+, specified below) are illustrated by the program in Figure 1, where we use Java-like syntax (we will use this syntax as long as it translates in a straightforward way to a Jinja/Jinja+ syntax).

Jinja comes equipped with a type system and a notion of well-typed programs. In this paper we consider only welltyped programs.

Semantics. Following [23], we briefly sketch the small-step semantics of Jinja. The full set of rules, including those for Jinja+ (see the next subsection) can be found in Appendix B.

A state is a pair of heap and a store. A store is a map from variable names to values. A heap is a map from references (addresses) to object instances. An object instance is a pair consisting of a class name and a field table, and a field table is a map from field names (which include the class where a field is defined) to values.

The small-step semantics of Jinja is given as a set of rules of the form $P \vdash\langle e, s\rangle \rightarrow\left\langle e^{\prime}, s^{\prime}\right\rangle$, describing a single step of the program execution (reduction of an expression). We will call $\langle e, s\rangle\left(\left\langle e^{\prime}, s^{\prime}\right\rangle\right)$ a configuration. In this rule, $P$ is a program in the context of which the evaluation is carried out, $e$ and $e^{\prime}$ are expressions and $s$ and $s^{\prime}$ are states. Such a rule says that, given a program $P$ and a state $s$, an expression $e$ can be reduced in one step to $e^{\prime}$, changing the state to $s^{\prime}$.

\section{B. Jinja+}

As a basis of our formal results we take a language that extends Jinja with: (a) the primitive type byte with natural conversions from and to int, (b) arrays, (c) abort primitive, (d) static fields (with the restriction that they can be initialized by literals only), (e) static methods, (f) access modifier for classes, fields, and methods (such as private, protected, and public), (g) final classes (classes that cannot be extended), (h) the throws clause of a method declaration.

Exceptions, which are already part of Jinja, are particularly critical for the security properties we are interested in because they provide an additional way information can be transfered from one part of the program to another.

We assume that Jinja+ programs have unbounded memory. The reason for this modeling choice is that the formal foundation for the security notions adopted in this paper are based on asymptotic security. This kind of security definitions only makes sense if the memory is not bounded, since the security parameter grows indefinitely.

Randomized programs. So far, we have considered deterministic programs. We will also need to consider randomized programs in our framework. For this purpose, Jinja+ programs may use the primitive randomBit() that returns a random bit each time it is used. Jinja+ programs that do not make use of randomBit ( ) are (called) deterministic, and otherwise, they are called randomized.

An example of a Jinja+ program is, as mentioned, presented in Figure 1.

Runs of Jinja+ programs. As already mentioned, the full set of rules of the small-step semantics of Jinja+ can be found in Appendix B. Based on this small-step semantics, we now define runs of Jinja+ programs.

Definition 1. A run of a deterministic program $P$ is a sequence of configurations obtained using the (small-step) Jinja+ semantics from the initial configuration of the form $\left\langle e_{0},\left(h_{0}, l_{0}\right)\right\rangle$, where $e_{0}=C$. main (), for $C$ being the (unique) class where main is defined, $h_{0}=\emptyset$ is the empty heap, $l_{0}$ is the store mapping the static (global) variables to their initial values (if the initial value for a static variable is not specified in the program, the default initial value for its type is used).

A randomized program induces a distribution of runs in the obvious way. Formally, such a program is a random variable from the set $\{0,1\}^{\omega}$ of infinite bit strings into the set of runs (of deterministic programs), with the usual probability space over $\{0,1\}^{\omega}$, where one infinite bit string determines the outcome of randomBit(), and hence, induces exactly one run.

The small-step semantics of Jinja+ provides a natural measure for the length of a run of a program, and hence, the 


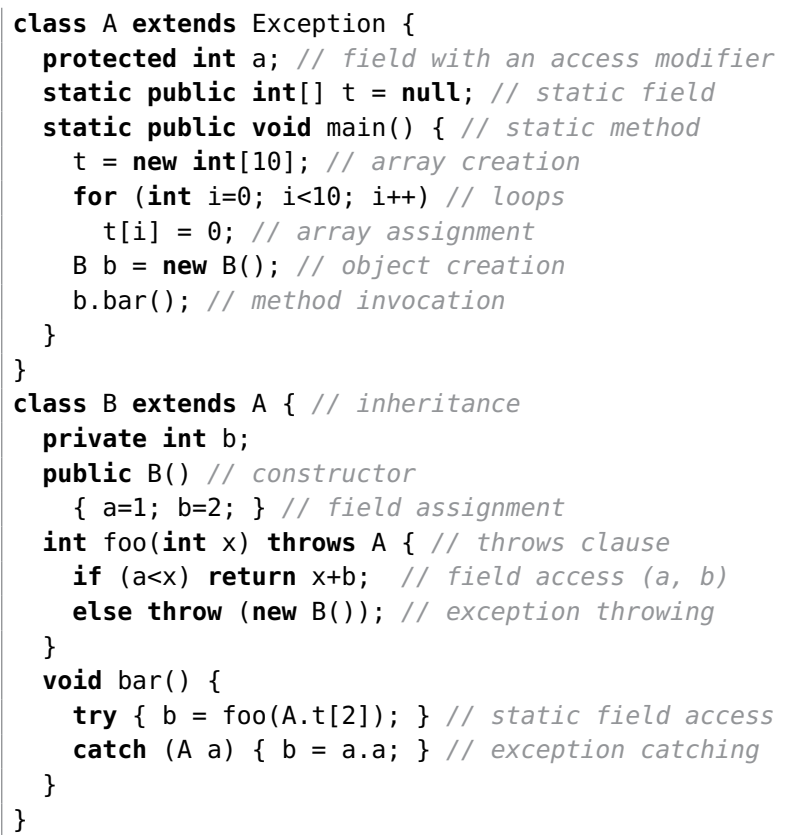

Figure 1. An example Jinja+ program (in Java-like notation).

runtime of a program. The length of a run of a deterministic program is the number of steps taken using the rules of the small-step semantics. Given this definition, for a randomized program the length of a run is a random variable defined in the obvious way.

For a run $r$ of a program $P$ containing some subprogram $S$ (a subset of classes of $P$ ), we define the number of steps performed by $S$ or the number of steps performed in the code of $S$ in the expected way. To define this notion, we keep track of the origin of (sub)expressions, i.e., the class they come from. If a rule is applied on a (sub)expression that originates from the class $C$, we label this step with $C$ and count this as a step performed in $C$ (see Appendix B-B for details).

\section{INDISTINGUISHABILITY}

We now define what it means for two systems to be indistinguishable by environments interacting with those systems. Indistinguishability is a fundamental relationship between systems which is interesting in its own right, for example, to define privacy properties, and to define simulation-based security, as we will see in the subsequent sections.

For this purpose, we first define interfaces that systems use/provide, how systems are composed, and environments. We then define the two forms of indistinguishability already mentioned in the introduction, namely perfect and computational indistinguishability. Since we consider asymptotic security, this involves to define programs that take a security parameter as input and that run in polynomial time in the security parameter.
Our definitions of indistinguishability follow the spirit of definitions of (computational) indistinguishability in the cryptographic literature (see, e.g., [10], [24], [21]), but, of course, instead of interactive Turing machines, we consider Jinja+ systems/programs. In particular, the simple communication model based on tapes is replaced by rich objectoriented interfaces between subsystems.

\section{A. Interfaces and Composition}

Before we define the notion of an interface, we emphasize that it should not be confused with the concept of interfaces in Java; we use this term with a different meaning.

Definition 2. An interface I is defined like a (Jinja+) system but where all method bodies as well as static field initializers are dropped.

If $I$ and $I^{\prime}$ are interfaces, then $I^{\prime}$ is a subinterface of $I$, written $I^{\prime} \sqsubseteq I$, if $I^{\prime}$ can be obtained from $I$ by dropping whole classes (with their method and field declarations), dropping methods and fields, dropping extends clauses, and/or adding the final modifier to class declarations.

Two interfaces are called disjoint if the set of class names declared in these interfaces are disjoint.

If $S$ is a system, then the public interface of $S$ is obtained from $S$ by (1) dropping all private fields and methods from $S$ and (2) dropping all method bodies and initializers of static fields.

Definition 3. A system $S$ implements an interface $I$, written $S: I$, if $I$ is a subinterface of the public interface of $S$.

Clearly, for every system $S$ we have that $S: \emptyset$.

Definition 4. We say that a system $S$ uses an interface I, written $I \vdash S$, if, besides its own classes, $S$ uses at most classes/methods/fields declared in $I$. We always assume that the public interface of $S$ and $I$ are disjoint.

We note that if $I \sqsubseteq I^{\prime}$ and $I \vdash S$, then $I^{\prime} \vdash S$. We write $I_{0} \vdash S: I_{1}$ for $I_{0} \vdash S$ and $S: I_{1}$. If $I=\emptyset$, i.e., $I$ is the empty interface, we often write $\vdash S$ instead of $\emptyset \vdash S$. Note that $\vdash S$ means that $S$ is a complete program.

Definition 5. Interfaces $I_{1}$ and $I_{2}$ are compatible if there exists an interface $I$ such that $I_{1} \sqsubseteq I$ and $I_{2} \sqsubseteq I$.

Intuitively, if two compatible interfaces contain the same class, the declarations of methods and fields of this class in those interfaces must be consistent (for instance, a field with the same name, if declared in both interfaces, must have the same type). Note that if $I_{1}$ and $I_{2}$ are disjoint, then they are compatible. Systems that use compatible interfaces and implement disjoint interfaces can be composed:

Definition 6 (Composition). Let $I_{S}, I_{T}, I_{S}^{\prime}$ and $I_{T}^{\prime}$ be interfaces such that $I_{S}$ and $I_{T}$ are disjoint and $I_{S}^{\prime}$ and $I_{T}^{\prime}$ are compatible. Let $S$ and $T$ be systems such that not both $S$ 
and $T$ contain the method main, $I_{S}^{\prime} \vdash S: I_{S}$, and $I_{T}^{\prime} \vdash T: I_{T}$. Then, we say that $S$ and $T$ are composable and denote by $S \cdot T$ the composition of $S$ and $T$ which, formally, is the union of (declarations in) $S$ and $T$. If the same classes are defined both in $S$ and $T$ (which may happen for classes not specified in $I_{S}$ and $I_{T}$ ), then we always implicitly assume that these classes are renamed consistently in order to avoid name clashes.

We emphasize that the interfaces between subsystems as considered here are quite different and much richer than the interfaces between interactive Turing machines considered in cryptography. Instead of plain bit strings that are sent over tapes between different machines, objects can be created, classes of another subsystem can be extended by inheritance, and data of different types, including references pointing to possibly complex objects, can be passed between different objects. Also, the flow of control is different. While in the Turing machine model, sending a message gives control to the receiver of the message and this control might not come back to the sender, in the object-oriented setting communication goes through method calls and fields. After a method call, control comes back to the caller, provided that potential exceptions are caught and the execution is not aborted.

We also emphasize that while a setting of the form $\vdash S: I$ and $I \vdash T$, i.e., in $S \cdot T$ the system $T$ uses the interface $I$ implemented by $S$, suggests that the initiative of accessing fields and calling methods always comes from $T$, it might also come from $S$ by using callback objects: $T$ could extend classes of $S$ by inheritance, create objects of these classes and pass references to these objects to $S$ (by calling methods of $S$ ). Then, via these references, $S$ can call methods specified in $T$. (This, in fact, is a common programming technique.)

\section{B. Environments}

An environment will interact with one of two systems and it has to decide with which system it interacted (see Section III). Its decision is written to a distinct static boolean variable result.

Definition 7. A system $E$ is called an environment if it declares a distinct private static variable result of type boolean with initial value false.

In the rest of the paper, we (often implicitly) assume that the variable result is unique in every Java program, i.e., it is declared in at most one class of a program, namely, one that belongs to the environment.

Definition 8. Let $S$ be a system with $S: I$ for some interface $I$. Then an environment $E$ is called an I-environment for $S$ if there exists an interface $I_{E}$ disjoint from $I$ such that (i) $I_{E} \vdash S: I$ and $I \vdash E: I_{E}$ and (ii) either $S$ or $E$ contains main().
Note that $E$ and $S$, as in the above definition, are composable and $E \cdot S$ is a (complete) program.

For a finite run of $E \cdot S$, i.e., a run that terminates, we call the value of result at the end of the run the output of $E$ or the output of the program $E \cdot S$. For infinite runs, we define the output to be false. If $E \cdot S$ is a deterministic program, then we write $E \cdot S \rightsquigarrow$ true if the output of $E \cdot S$ is true. If $E \cdot S$ is a randomized program, we write $\operatorname{Prob}\{E \cdot S \rightsquigarrow$ true $\}$ to denote the probability that the output of $E \cdot S$ is true.

Definition 9 (same interface). The systems $S_{1}$ and $S_{2}$ use the same interface if (i) for every $I_{E}$, we have that $I_{E} \vdash S_{1}$ iff $I_{E} \vdash S_{2}$, and (ii) $S_{1}$ contains the method main iff $S_{2}$ contains main.

Observe that if $S_{1}$ and $S_{2}$ use the same interface and we have that $S_{1}: I$ and $S_{2}: I$ for some interface $I$, then every $I$-environment for $S_{1}$ is also an $I$-environment for $S_{2}$.

\section{Programs with security parameter}

As mentioned at the beginning of this section, we need to consider programs that take a security parameter as input and run in polynomial time in this security parameter.

To ensure that all parts of a system have access to the security parameter, we fix a distinct interface $I_{S P}$ consisting of (one class containing) one public static variable securityParameter. We assume that, in all the considered systems/programs, this variable (after being initialized) is only read but never written to. Therefore, all parts of the considered system can, at any time, access the same, initial value of this variable.

For a natural number $\eta$, we define a system $S P_{\eta}$ that implements the interface $I_{S P}$ by setting the initial value of securityParameter to $\eta$. We do not fix here how this value is represented because the representation is not essential for our results; it could be represented as a linked list of objects or an array (see also the discussion below).

We call a system $P$ such that $I_{S P} \vdash P$ a program with a security parameter or simply a program if the presence of a security parameter is clear from the context. Note that by this, $S P_{\eta} \cdot P$ is a complete program, which we abbreviate by $P(\eta)$.

Although as far as asymptotic security is concerned, our framework works fine with the definitions we have introduced so far, they are not perfectly aligned with the common practice of programming in Java. More specifically, messages, such as keys, ciphertexts, digital signatures, etc., are typically represented as arrays of bytes. However, this representation is bounded by the maximal length of an array, which is the maximal value of an integer (int). Therefore, following common programming practice, there would be a strict bound on, for example, the maximal size of keys (if represented as arrays of bytes). Since we consider asymptotic security, the size of keys should, however, grow with the security parameter. 
One solution could be to use another representation of messages, such as lists of bytes. This, however, would result in unnatural programs and we, of course, want to be able to analyze programs as given in practice. Another solution could be to use concrete instead of asymptotic security definitions. However, most results in simulationbased security are formulated w.r.t. asymptotic security, and hence, we would not be able to reuse these results and avoid, for example, reproving from scratch realizations of ideal functionalities.

Therefore, we prefer the following solution. We parameterize the semantics of Jinja+ with the maximal (absolute) value integers can take. So if $P$ is a deterministic program, the run of $P$ with integer size $s \geq 1$ is a run of $P$ where the maximal (absolute) value of integers is $s$; analogously for randomized programs. We write $P \rightsquigarrow_{s}$ true if the output of such a run is true; analogously, we define $\operatorname{Prob}\left\{P \rightsquigarrow_{s}\right.$ true $\}$. In our asymptotic formulations of indistinguishability, the size of integers may depend on the security parameter.

\section{Perfect Indistinguishability}

We now formulate perfect indistinguishability, which, as we will prove in Section V, implies computational indistinguishability. We say that a deterministic program $P$ terminates for integer size $s$, if the run of $P$ with integer size $s$ is finite.

Definition 10 (Perfect indistinguishability). Let $S_{1}$ and $S_{2}$ be deterministic systems with a security parameter and such that $S_{1}: I$ and $S_{2}: I$ for some interface $I$. Then, $S_{1}$ and $S_{2}$ are perfectly indistinguishable w.r.t. I, written $S_{1} \approx_{\text {perf }}^{I} S_{2}$, if (i) $S_{1}$ and $S_{2}$ use the same interface and (ii) for every deterministic $I$-environment $E$ for $S_{1}$ (and hence, $S_{2}$ ) with security parameter, for every security parameter $\eta$ and every integer size $s \geq 1$, it holds that if $E \cdot S_{1}(\eta)$ and $E \cdot S_{2}(\eta)$ terminate for integer size $s$, then $E \cdot S_{1}(\eta) \rightsquigarrow_{s}$ true iff $E$. $S_{2}(\eta) \rightsquigarrow_{s}$ true.

We note that the notion of perfect indistinguishability introduced above is termination-insensitive, i.e. it puts no restrictions on non-terminating runs. This (weak) form of perfect indistinguishability, nevertheless implies computational indistinguishability (see Theorem 3).

\section{E. Polynomially Bounded Systems}

As already mentioned at the beginning of this section, in order to define the notion of computational indistinguishability we need to define programs and environments whose runtime is polynomially bounded in the security parameter.

For this purpose, we fix now and for the rest of this section a polynomially computable function intsize that takes a security parameter $\eta$ as input and outputs a natural number $\geq 1$. We require that the numbers returned by this function are bounded by a fixed polynomial in the security parameter.
All notions defined in what follows are parameterized by that function. However, due to ease of notion this will not be made explicit.

Our runtime definitions follow the spirit of definitions in cryptographic definitions of simulation-based security, in particular, [24], [21].

We start with the definition of almost bounded programs. These are programs that, with overwhelming probability, terminate after a polynomial number of steps.

Definition 11 (Almost bounded). A program $P$ with security parameter is almost bounded if there exists a polynomial $p$ such that the probability that the length of a run of $P(\eta)$ (with integer size intsize $(\eta)$ ) exceeds $p(\eta)$ is a negligible function in $\eta{ }^{1}$

It is easy to see that an almost bounded program $P$ can be simulated by a probabilistic polynomial time Turing machine that simulates at most $p(\eta)$ steps of a run of $P(\eta)$ (with integer size intsize $(\eta)$ ) and produces output that is distributed the same up to a negligible difference.

We also need the notion of a bounded environment. The number of steps such an environment performs in a run is bounded by a fixed polynomial independently of the system the environment interacts with.

Definition 12 (Bounded environment). An environment $E$ is called bounded if there exists a polynomial $p$ such that, for every system $S$ such that $E$ is an $I$-environment for $S$ (for some interface $I$ ) and for every run of $E \cdot S(\eta$ ) (with integer size intsize $(\eta)$ ), the number of steps performed in the code of $E$ does not exceed $p(\eta)$.

This definition makes sense since $E$ can abort a run by calling abort(), and hence, $E$ can prevent to be called by $S$, which would always require to execute some code in $E$. (Without abort(), $E$ can, in general, not prevent that code fragments of $E$ are executed, e.g., $S$ could keep calling methods of classes of $E$.)

If an environment $E$ is both bounded and an $I$-environment for some system $S$, we call $E$ a bounded I-environment for $S$.

For the cryptographic analysis of systems to be meaningful, we study systems that run in polynomial time (with overwhelming probability) with any bounded environment.

Definition 13 (Environmentally $I$-bounded). A system $S$ is environmentally I-bounded, if $S: I$ and for each bounded $I$-environment $E$ for $S$, the program $E \cdot S$ is almost bounded.

It is typically easy to see that a system is environmentally $I$-bounded (for all functions intsize).

We note that environmentally $I$-bounded systems, as defined above, are reactive systems that are free to process

\footnotetext{
${ }^{1}$ As usual, a function $f$ from the natural numbers to the real numbers is negligible, if for every $c>0$ there exists $\eta_{0}$ such that $f(\eta) \leq \frac{1}{\eta^{c}}$ for all $\eta>\eta_{0}$. A function $f$ is overwhelming if 1-f is negligible.
} 
an unbounded number of requests of the environment $E$. In particular, a reactive system $S$ does not need to terminate after some fixed and bounded number of requests. Clearly, every bounded $I$-environment, being bounded, will only invoke $S$ a bounded number of times, more precisely, the number of invocations the environment makes is bounded by some polynomial in the security parameter.

\section{F. Computational Indistinguishability}

Having defined polynomially bounded systems and programs, we are now ready to define computational indistinguishability of systems, where, again, we fix the function intsize. (However, computational guarantees for Java programs will be independent of a specific function intsize.) We start with the notion of computationally equivalent programs.

Definition 14 (Computational Equivalence). Let $P_{1}$ and $P_{2}$ be (complete, possibly probabilistic) programs with security parameter. Then $P_{1}$ and $P_{2}$ are computationally equivalent, written $P_{1} \equiv_{\text {comp }} P_{2}$, if $\mid \operatorname{Prob}\left\{P_{1}(\eta) \rightsquigarrow\right.$ intsize $(\eta)$ true $\}-$ $\operatorname{Prob}\left\{P_{2}(\eta) \rightsquigarrow\right.$ intsize $(\eta)$ true $\} \mid$ is a negligible function in the security parameter $\eta$.

Definition 15 (Computational indistinguishability). Let $S_{1}$ and $S_{2}$ be environmentally $I$-bounded systems. Then $S_{1}$ and $S_{2}$ are computationally indistinguishable w.r.t. I, written $S_{1} \approx_{\text {comp }}^{I} S_{2}$, if $S_{1}$ and $S_{2}$ use the same interface and for every bounded $I$-environment $E$ for $S_{1}$ (and hence, $S_{2}$ ) we have that $E \cdot S_{1} \equiv_{\text {comp }} E \cdot S_{2}$.

Typically, this definition is applied to systems $S_{1}$ and $S_{2}$ that do not use the statement abort(). However, our results also work in this case.

We also note that this definition of indistinguishability is w.r.t. uniform environments. A definition w.r.t. non-uniform environments can be obtained in a straightforward way by giving the environment additional auxiliary input (besides the security parameter).

Furthermore, we point out that in the above definition two cases can occur: (1) main() is defined in $E$ or (2) main() is defined in both $S_{1}$ and $S_{2}$. In the first case, $E$ can freely create objects of classes in the interface $I$ (which is a subset of classes of $S_{1} / S_{2}$ ) and initiate calls. Eventually, even in case of exceptions, $E$ can get back control (method calls return a value to $E$ and $E$ can catch exceptions if necessary), unless $S_{1} / S_{2}$ uses abort. The kind of control $E$ has in the case (2), heavily depends on the specification of $S_{1} / S_{2}$. This can go from having as much control as in case (1) to being basically a passive observer. For example, main() (as specified in $\left.S_{1} / S_{2}\right)$ could call a method of $E$ and from then on $E$ can use the possibly very rich interface $I$ as in case (1). The other extreme is that $I$ is empty, say, so $E$ cannot create objects of (classes of) $S_{1} / S_{2}$ by itself, only $S_{1} / S_{2}$ can create objects of (classes of) $E$ and of $S_{1} / S_{2}$. Hence, $S_{1} / S_{2}$ has more control and can decide, for instance, how many and which objects are created and when $E$ is contacted. Still even in this case, if so specified, $S_{1} / S_{2}$ could give $E$ basically full control by callback objects (see Section III-A). (As a side note, illustrating the richness of the interfaces, compared to Turing machine models, $E$ could also extend classes of $S_{1} / S_{2}$ and by this, if not properly protected, might get access to information kept in these classes.)

\section{Simulatability}

We now formulate what it means for a system to realize another system, in the spirit of the simulation-based approach.

As before, we fix a function intsize (see Section III-E) for the rest of this section. Typically, one would prove that one system realizes the other for all such functions.

Our formulation of the realization of one system by another follows the spirit of strong simulatability in the simulation-based approach (see, e.g., [24]). In a nutshell, the definition says that the (real) system $R$ realizes an (ideal) system $F$ if there exists a simulator $S$ such that $R$ and $S \cdot F$ behave almost the same in every bounded environment.

Definition 16 (Strong Simulatability). Let $I_{\text {in }}, I_{\text {out }}, I_{E}, I_{S}$ be disjoint interfaces. Let $F$ and $R$ be systems. Then $R$ realizes $F$ w.r.t. the interfaces $I_{\text {out }}, I_{i n}, I_{E}$, and $I_{S}$, written $R \leq{ }^{\left(I_{\text {out }}, I_{\text {in }}, I_{E}, I_{S}\right)} F$ or simply $R \leq F$, if i) $I_{E} \cup I_{\text {in }} \vdash R: I_{\text {out }}$ and $I_{E} \cup I_{\text {in }} \cup I_{S} \vdash F: I_{\text {out }}$, ii) either both $F$ and $R$ or neither of these systems contain the method main(), iii) $R$ is an environmentally $I_{\text {out }}$-bounded system ( $F$ does not need to be), and iv) there exists a system $S$ (the simulator) such that $S$ does not contain main(), $I_{E} \vdash S: I_{S}, S \cdot F$ is environmentally $I_{\text {out }}$-bounded, and $R \approx_{\text {comp }}^{I_{\text {out }}} S \cdot F$.

The intuition behind the way the interfaces between the different components (environment, ideal and real functionalities, simulator) are defined is as follows: Both $R$ and $F$ provide the same kind of functionality/service, specified by the interface $I_{\text {out }}$. They may require some (trusted) services $I_{\text {in }}$ from another system component and some services $I_{E}$ from an (untrusted) environment, for example, networking and certain other libraries. In addition, the ideal functionality $F$ may require services $I_{S}$ from the simulator $S$, which in turn may require services $I_{E}$ from the environment. Recall from the discussion in Section III-A that the interfaces can be very rich-they allow for communication and method calls in both directions.

In the applications we envision, with our case study being the first example, $F$ will typically be an ideal functionality for one or more cryptographic primitives and its realization $R$ will basically be the actual cryptographic schemes.

The notion of strong simulatability, as introduced above, enjoys important basic properties, namely, reflexivity and transitivity, and allows to prove a fundamental composition theorem. To prove these results, we need the following lemma, with the proof provided in Appendix C-A. 
Lemma 1. Let $I_{E}$ and I be disjoint interfaces and let $S_{1}$ and $S_{2}$ be environmentally I-bounded systems such that $S_{1} \approx_{\text {comp }}^{I}$ $S_{2}$ (in particular, $S_{1}$ and $S_{2}$ use the same interface) and $I_{E} \vdash S_{1}: I$, and hence, $I_{E} \vdash S_{2}: I$. Let $E$ be a (not necessarily bounded) I-environment for $S_{1}$ (and hence, $S_{2}$ ) with $I \vdash E: I_{E}$ such that $E \cdot S_{1}$ is almost bounded. Then $E \cdot S_{2}$ is almost bounded and $E \cdot S_{1} \equiv_{\text {comp }} E \cdot S_{2}$.

Now we can prove reflexivity and transitivity of strong simulatability.

Lemma 2 (Reflexivity of strong simulatability). Let $I_{\text {out }}$, $I_{\text {in }}$, and $I_{E}$ be disjoint interfaces and let $R$ be a system such that $I_{E} \cup I_{\text {in }} \vdash R: I_{\text {out }}$ and $R$ is environmentally $I_{\text {out }}$-bounded. Then, $R \leq R$, i.e., $R$ realizes itself.

Proof: We define $S=\emptyset$, i.e., $S$ does not contain any class, and immediately obtain that $R \approx_{\text {comp }}^{I_{\text {out }}} R=S \cdot R$.

Lemma 3 (Transitivity of strong simulatability). Let $I_{\text {out }}$, $I_{\text {in }}, I_{E}, I_{S}^{0}$, and $I_{S}^{1}$ be disjoint interfaces and let $R_{0}, R_{1}$, and $R_{2}$ be environmentally I-bounded systems. If $R_{1} \leq\left(I_{\text {out }}, I_{\text {in }}, I_{E} \cup I_{S}^{1}, I_{S}^{0}\right)$ $R_{0}$ and $R_{2} \leq^{\left(I_{\text {out }}, I_{\text {in }}, I_{E}, I_{S}^{1}\right)} R_{1}$, then $R_{2} \leq^{\left(I_{\text {out }}, I_{\text {in }}, I_{E}, I_{S}^{0} \cup I_{S}^{1}\right)} R_{0}$.

Proof: Under the assumption of the lemma, we know that there exist $S_{0}$ and $S_{1}$ such that $I_{E} \cup I_{S}^{1} \vdash S_{0}: I_{S}^{0}, I_{E} \vdash S_{1}$ : $I_{S}^{1}, S_{0} \cdot R_{0}$ and $S_{1} \cdot R_{1}$ are environmentally $I_{\text {out }}$-bounded, and $R_{1} \approx_{\text {comp }}^{I_{\text {out }}} S_{0} \cdot R_{0}$ and $R_{2} \approx_{\text {comp }}^{I_{\text {out }}} S_{1} \cdot R_{1}$. We define $S=S_{0} \cdot S_{1}$. Obviously, we have that $I_{E} \vdash S: I_{S}^{0} \cup I_{S}^{1}$. Now let $E$ be a bounded $I_{\text {out }}$-environment for $R_{2}$. Then, we obtain:

$$
E \cdot R_{2} \equiv E \cdot S_{1} \cdot R_{1} \equiv E \cdot S_{1} \cdot S_{0} \cdot R_{0}=E \cdot S \cdot R_{0} .
$$

The first equivalence holds because of our assumptions. For the second equivalence, first note that $E \cdot S_{1} \cdot R_{1}$ is almost bounded and $R_{1} \approx_{\text {comp }}^{I_{\text {out }}} S_{0} \cdot R_{0}$. By Lemma 1, we now obtain that $E \cdot S_{1} \cdot S_{0} \cdot R_{0}$ is almost bounded and that the second equivalence holds.

In short, the following composition theorem says that if $R_{0}$ realizes $F_{0}$ and $R_{1}$ realizes $F_{1}$, then the composed real system $R_{0} \cdot R_{1}$ realizes the composed ideal system $F_{0} \cdot F_{1}$. In other words, it suffices to prove the realizations separately in order to obtain security of the composed systems.

Theorem 1 (Composition Theorem). Let $I_{0}, I_{1}, I_{E}, I_{S}^{0}$, and $I_{S}^{1}$ be disjoint interfaces and let $R_{0}, F_{0}, R_{1}$, and $F_{1}$ be systems such that $R_{0} \leq^{\left(I_{0}, I_{1}, I_{E}, I_{S}^{0}\right)} F_{0}, R_{1} \leq^{\left(I_{1}, I_{0}, I_{E}, I_{S}^{1}\right)} F_{1}$, not both $R_{0}$ and $R_{1}$ contain main (), and $R_{0} \cdot R_{1}$ are environmentally $\left(I_{0} \cup\right.$ $\left.I_{1}\right)$-bounded. Then, $R_{0} \cdot R_{1} \leq{ }^{\left(I_{0} \cup I_{1}, \emptyset, I_{E}, I_{S}^{0} \cup I_{S}^{1}\right)} F_{0} \cdot F_{1}$.

Proof: Under the assumptions of the theorem, there exist $S_{0}$ and $S_{1}$ such that $I_{E} \vdash S_{i}: I_{S}^{i}, S_{i} \cdot F_{i}$ is environmentally $I_{i}$-bounded and $R_{i} \approx_{\text {comp }}^{I_{i}} S_{i} \cdot F_{i}$ for $i \in\{0,1\}$.

We define $S=S_{0} \cdot S_{1}, R=R_{0} \cdot R_{1}, F=F_{0} \cdot F_{1}, I=I_{0} \cup I_{1}$, and $I_{S}=I_{S}^{0} \cup I_{S}^{1}$. In order to show that $R \leq \leq^{\left(I, \emptyset, I_{E}, I_{S}\right)} F$ it suffices to prove that a) $S \cdot F$ is environmentally $I$-bounded and b) $R \approx_{\text {comp }}^{I} S \cdot F$; the remaining conditions are obvious.
Let $E$ be a bounded $I$-environment for $R$. Similarly to the proof of Lemma 3, by Lemma 1 we obtain the following equivalences:

$$
\begin{aligned}
E \cdot R & =E \cdot R_{0} \cdot R_{1} \\
& \equiv E \cdot R_{0} \cdot\left(S_{1} \cdot F_{1}\right) \\
& \equiv E \cdot\left(S_{0} \cdot F_{0}\right) \cdot\left(S_{1} \cdot F_{1}\right)=E \cdot S \cdot F,
\end{aligned}
$$

This implies b). By Lemma 1 we, in particular, get that all the above systems are almost bounded. Since we quantified over all bounded $I$-environments for $R$ (and hence, $S \cdot F$ ) it follows that $S \cdot F$ is environmentally $I$-bounded, thus a).

Note that with $R_{0} \cdot R_{1} \leq^{\left(I_{0} \cup I_{1}, \emptyset, I_{E}, I_{S}^{0} \cup I_{S}^{1}\right)} F_{0} \cdot F_{1}$ we that $R_{0}$. $R_{1}$ realizes $F_{0} \cdot F_{1}$ also for all subinterfaces of $I_{0} \cup I_{1}$.

For simplicity, Theorem 1 is stated in such a way that the trusted service that $R_{i}$ may use is completely provided by $R_{i-1}$, namely through $I_{i-1}$. It is straightforward (only heavy in notation) to state and prove the theorem for the more general case that the trusted service is only partially provided by the other system.

\section{From Perfect to Computational INDISTINGUISHABILITY}

We now prove that if two systems that use an ideal functionality are perfectly indistinguishable, then these systems are computationally indistinguishable if the ideal functionality is replaced by its realization. As already explained in the introduction, this is a central step in enabling program analysis tools that cannot deal with cryptography and probabilistic polynomially bounded adversaries to establish computational indistinguishability properties. As before, we fix a function intsize (see Section III-E) for the rest of this section.

The proof is done via two theorems. The first says that if two systems that use an ideal functionality are computationally indistinguishable, then they are also computationally indistinguishable if the ideal functionality is replaced by its realization.

Theorem 2. Let I, J, $I_{E}, I_{S}$, and $I_{P}$ be disjoint interfaces with $J \sqsubseteq I_{P} \cup I$. Let $F, R, P_{1}$, and $P_{2}$ be systems such that (i) $I_{E} \cup I \vdash P_{1}: I_{P}$ and $I_{E} \cup I \vdash P_{2}: I_{P}$, (ii) $R \leq^{\left(I, I_{P}, I_{E}, I_{S}\right)} F$, in particular, $I_{E} \cup I_{P} \vdash R: I$ and $I_{E} \cup I_{P} \cup I_{S} \vdash F: I$, (iii) $P_{1}$ contains main() iff $P_{2}$ contains main(), (iv) not both $P_{1}$ and $F$ (and hence, $R$ ) contain main(), (v) $F \cdot P_{i}$ and $R \cdot P_{i}$, for $i \in$ $\{1,2\}$, are environmentally $J$-bounded. Then, $F \cdot P_{1} \approx_{\text {comp }}^{J}$ $F \cdot P_{2}$ implies $R \cdot P_{1} \approx_{\text {comp }}^{J} R \cdot P_{2}$.

Proof: Assume that $F \cdot P_{1} \approx_{\text {comp }}^{J} F \cdot P_{2}$. In particular, $F \cdot P_{1}$ and $F \cdot P_{2}$ use the same interface and, therefore $R \cdot P_{1}$ and $R \cdot P_{2}$ use the same interface as well.

Let $E$ be a bounded $J$-environment for $R \cdot P_{1}$. We need to show that $E \cdot R \cdot P_{1} \equiv_{\text {comp }} E \cdot R \cdot P_{2}$. 
Because $R \leq^{\left(I, I_{P}, I_{E}, I_{S}\right)} F$, there exists a simulator $S$ such that $I_{E} \vdash S: I_{S}, S \cdot F$ is environmentally $I$-bounded and

$$
R \approx_{\text {comp }}^{I} S \cdot F
$$

Now, because $R \cdot P_{i}, i \in\{1,2\}$, is environmentally $J$-bounded, the system $E \cdot R \cdot P_{i}$ is almost bounded. By (1) and Lemma 1 we can conclude that $E \cdot S \cdot F \cdot P_{i}$ is almost bounded and

$$
E \cdot R \cdot P_{i} \equiv_{\mathrm{comp}} E \cdot S \cdot F \cdot P_{i} .
$$

As we have assumed that $F \cdot P_{1} \approx_{\text {comp }}^{J} F \cdot P_{2}$, by Lemma 1 we obtain

$$
E \cdot S \cdot F \cdot P_{1} \equiv_{\mathrm{comp}} E \cdot S \cdot F \cdot P_{2} .
$$

Combining (2) and (3), we obtain $E \cdot R \cdot P_{1} \equiv_{\mathrm{comp}} E \cdot R \cdot P_{2}$.

For simplicity of presentation, the theorem is formulated in such a way that $P_{i}, i \in\{1,2\}$, uses only $I$ as a (trusted) service and $F / R$ uses $I_{P}$. It is straightforward to also allow for other external services.

We now show that perfect indistinguishability implies computational indistinguishability (see Appendix C-B) for the proof.

Theorem 3. Let I be an interface and let $S_{1}$ and $S_{2}$ be deterministic, environmentally I-bounded programs such that $S_{i}: I$, for $i \in\{1,2\}$, and $S_{1}$ and $S_{2}$ use the same interface. Then, $S_{1} \approx_{\text {perf }}^{I} S_{2}$ implies $S_{1} \approx_{\text {comp }}^{I} S_{2}$.

By combining Theorem 2 and Theorem 3, we obtain the desired result explained at the beginning of this section.

Corollary 1. Under the assumption of Theorem 2 and moreover assuming that $P_{1} \cdot F$ and $P_{2} \cdot F$ are deterministic systems, it follows that $P_{1} \cdot F \approx_{\text {perf }}^{J} P_{2} \cdot F$ implies $P_{1} \cdot R \approx_{\text {comp }}^{J} P_{2} \cdot R$.

Recall that $P_{1} \cdot R \approx_{\text {comp }}^{J} P_{2} \cdot R$ is (implicitly) defined w.r.t. the integer size function intsize $(\eta)$. However, since the statement $P_{1} \cdot F \approx_{\text {perf }}^{J} P_{2} \cdot F$ does not depend on any integer size function, we obtain that computational indistinguishability holds independently of a specific integer size function.

\section{Perfect Indistinguishability AND NON-INTERFERENCE}

In this section we prove that perfect indistinguishability and non-interference are equivalent. Hence, in combination with Corollary 1 it suffices for tools to analyze systems that use an ideal functionality w.r.t. non-interference in order to get computational indistinguishability for systems when the ideal functionality is replaced by its realization. As already mentioned in the introduction, many tools can analyze noninterference for Java programs.

The (standard) non-interference notion for confidentiality [17] requires the absence of information flow from high to low variables within a program. In this paper, we define non-interference for a (Jinja+) program $P$ with some static variables $\vec{x}$ of primitive types that are labeled as high. Also, some other static variables of primitive types are labeled as low. We say that $P[\vec{x}]$ is a program with high and low variables. By $P[\vec{a}]$ we denote the program $P$ where the high variables $\vec{x}$ are initialized with values $\vec{a}$ and the low variables are initialized as specified in $P$. We assume that the length of $\vec{x}$ and $\vec{a}$ are the same and $\vec{a}$ contains values of appropriate types; in such a case we say that $\vec{a}$ is valid. Now, non-interference for a (deterministic) program is defined as follows.

Definition 17 (Non-interference for Jinja+ programs). Let $P[\vec{x}]$ be a program with high and low variables. Then, $P[\vec{x}]$ has the non-interference property if the following holds: for all valid $\vec{a}_{1}$ and $\vec{a}_{2}$ and all integer sizes $s \geq 1$, if $P\left[\vec{a}_{1}\right]$ and $P\left[\vec{a}_{2}\right]$ terminate for integer size $s$, then at the end of these runs, the values of the low variables are the same.

Similarly to the definition of perfect indistinguishability (Definition 10), the above definition captures terminationinsensitive non-interference.

We note that the non-interference property is quite powerful: $P$ could have just one high variable of type boolean. Depending on the value of this variable $P$ could run one of two systems $S_{1}$ and $S_{2}$, illustrating that the non-interference property can be as powerful as perfect indistinguishability.

The above notion of non-interference deals with complete programs (closed systems). We now generalize this definition to open systems:

Definition 18 (Non-interference in an open system). Let $I$ be an interface and let $S[\vec{x}]$ be a (not necessarily closed) deterministic system with a security parameter, high and low variables, and such that $S: I$. Then, $S[\vec{x}]$ is I-noninterferent if for every deterministic $I$-environment $E$ for $S[\vec{x}]$ and every security parameter $\eta$ non-interference holds for the system $E \cdot S[\vec{x}](\eta)$, where the variable result declared in $E$ is considered to be a low variable.

Now, equivalence of this notion and perfect indistinguishability follows easily by the definitions of $I$-noninterference and perfect indistinguishability:

Theorem 4. Let I and $S[\vec{x}]$ be given as in Definition 18 with no variable of $S$ labeled as low (only the variable result declared in the environment is labeled as low). Then the following statements are equivalent:

(a) For all valid $\vec{a}_{1}$ and $\vec{a}_{2}$, we have that $S\left[\vec{a}_{1}\right] \approx_{\text {perf }}^{I} S\left[\vec{a}_{2}\right]$.

(b) I-non-interference holds for $S[\vec{x}]$.

As already explained in the introduction and at the beginning of this section, in combination with Corollary 1, this theorem reduces the problem of checking computational indistinguishability for systems that use real cryptographic schemes to checking non-interference for systems that use ideal functionalities. 


\section{A PRoof TeChNique For NON-INTERFERENCE IN OPEN SYSTEMS}

There are many tools that can deal with classical noninterference for closed systems, i.e., complete programs. In this section, we develop proof techniques that enable such tools, at least some of them, to also deal with open systems. Technically, we will show that in order to check non-interference for open systems according to Definition 18 it is sufficient to consider only a very restricted class of environments, rather than all environments. The input these environments give to the system they interact with is fixed for every environment and does not depend on the output the environment got from the system. In fact, the environments in this class are all almost identical, they only differ in the input sequence that they use. Now, the analysis a tool performs often ignores or can ignore specific values encoded in the program, such as the input sequence. So, if such an analysis establishes non-interference for a system and a fixed environment in the considered class, then this implies that non-interference holds for all environments in this class. By our proof technique, it then follows that non-interference holds for all environments, as required by Definition 18 .

In our case study, we demonstrate that using our proof techniques, the tool Joana, which is designed to check non-interference for closed systems, can now deal with (a relevant class of) open systems as well.

To illustrate the main idea, we start with a simple case where communication between the environment and the system is restricted to primitive types only. We then generalize the result to the case where the environment and the system can communicate using also exceptions, arrays of bytes, and simple objects.

\section{A. Communication through Primitive Types Only}

In this section, we assume that a system $S$ communicates with an environment $E$ only through static functions with primitive types. More precisely, we consider programs $S$ such that (1) method main is defined in $S$ and (2) $I_{E} \vdash S$, for some interface $I_{E}$, where all methods are static, use primitive types only (for simplicity of presentation we will consider only the type int), and have empty throws clause. We will consider indistinguishability w.r.t. the empty interface (i.e. environments we consider do not directly call $S$ ).

The above assumptions will allow us to show, in the proof of Theorem 5, that $E$ and $S$ do not share any references: their states are in this sense disjoint.

We now define the class of environments mentioned at the beginning of this section. We then show that to establish $I$-non-interference, it is enough to consider only those environments (see Theorem 5).

For a finite sequence $\vec{u}=u_{1}, \ldots, u_{n}$ of values of type int, we denote by $\tilde{E}_{\vec{u}}^{I_{E}}$ the following system.

The environment $\tilde{E}_{\vec{u}}^{I_{E}}$ contains two static methods: untrustedoutput and untrustedInput, as specified in Fig-

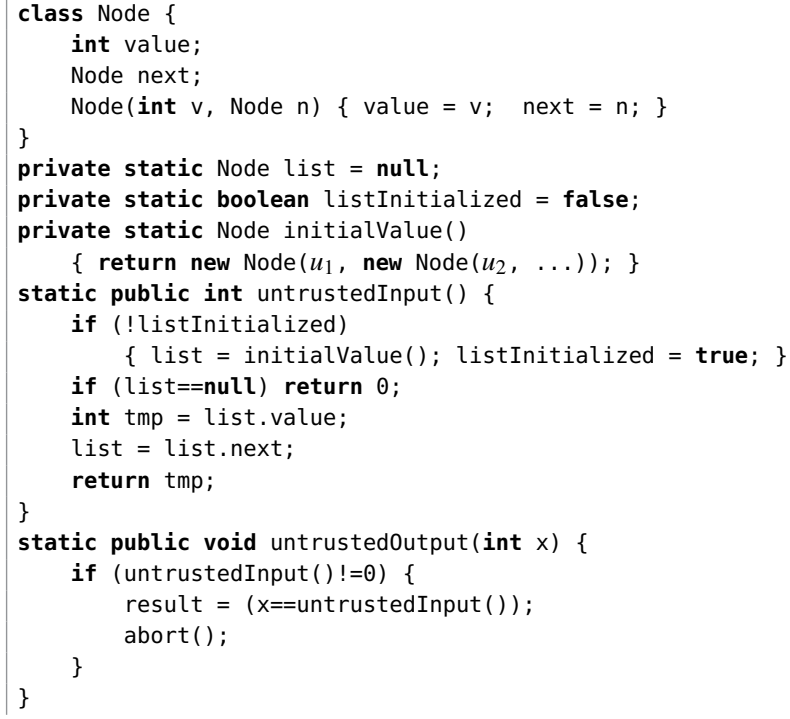

Figure 2. Implementation of untrustedInput and untrustedoutput in $\tilde{E}_{\vec{u}}$. We assume that class Node is not used anywhere else.

ure 2. For the sake of the discussion, let $S$ be the system the environment $\tilde{E}_{\vec{u}}^{I_{E}}$ interacts with. (Note that the definition of $\tilde{E}_{\vec{u}}^{I_{E}}$ is independent of a specific $S$. It only depends on $I_{E}$.) As we will see below, the method untrustedoutput gets all data passed by $S$ to $\tilde{E}_{\vec{u}}^{I_{E}}$. The method untrustedInput determines what the environment passes on to $S$.

More specifically. the method untrustedInput() returns consecutive values of $\vec{u}$ and, after the last element of $\vec{u}$ has been returned, it returns 0 . Note that the consecutive values returned by this method are hardwired in line 9 (determined by $\vec{u}$ ) and do not depend on any input to $\tilde{E}_{\vec{u}}^{I_{E}}$.

The method untrustedoutput, depending on the values given by untrustedInput (), either ignores its argument or compares its value to the next integer it obtains, again, from untrustedInput () and stores the result of this comparison in the (low) variable result. The intuition is the following: untrustedOutput gets, as we will see below, all the data the environment gets from $S$. If the two instances of $S$, $S\left[\vec{a}_{1}\right]$ and $S\left[\vec{a}_{2}\right]$, which the environment tries to distinguish, behave differently, then there must be some point where the environment gets different data from the two systems in the corresponding runs, i.e., untrustedOutput will be invoked with different values for $\mathrm{x}$, say the values $\mathrm{x}$ takes at this point are $b_{1}$ and $b_{2}$, respectively. By choosing an appropriate $\vec{u}$, this can be detected by untrustedOutput: $\vec{u}$ should be defined in such a way that the method untrustedInput() returns 1 at this point and that the value untrustedinput() returns next equals $b_{1}$, say ( $b_{2}$ would also work). Then, in the run of the environment with $S\left[\vec{a}_{1}\right]$ the variable result will be assigned 1 and in the run with $S\left[\vec{a}_{2}\right]$ it will be assigned 0 . Hence, the environment successfully distinguished $S\left[\vec{a}_{1}\right]$ 


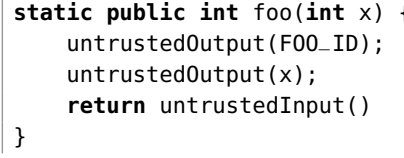

Figure 3. $\quad \tilde{E}_{\vec{u}}^{I_{E}}$ : the implementation of a method of $I_{E}$ with the signature static public int foo(int x), where F00_ID is an integer constant serving as the identifier of this method (we assign a different identifier to every method).

and $S\left[\vec{a}_{2}\right]$.

Finally, for every method declaration $m$ in $I_{E}$, the system $\tilde{E}_{\vec{u}}^{I_{E}}$ contains the implementation of $m$ as illustrated by the example in Figure 3. As we can see, the defined method forwards all its input data to untrusted0utput and lets untrustedInput determine the returned value.

This completes the definition of $\tilde{E}_{\vec{u}}^{I_{E}}$. The next theorem (see Appendix D-A for the proof) states that, to prove $I$-noninterference, it is enough to consider only environments $\tilde{E}_{\vec{u}}^{I_{E}}$ for all $\vec{u}$. In this way we need to study only (almost) closed systems, namely systems that differ in only one expression (line 9). As discussed at the beginning of this section, this restriction is often sufficient for tools are designed to deal with closed systems only.

Theorem 5. Let $I_{E}$ be an interfaces with only static methods of primitive argument and return types as introduced above. Let $S$ be a system with high and low variables such that main is defined in $S$ and $I_{E} \vdash S$. Then, I-non-interference, for $I=\emptyset$, holds for $S$ if and only if for all sequences $\vec{u}$ as above non-interference holds for $\tilde{E}_{u}^{I_{E}} \cdot S$.

\section{B. Communication through Arrays, Simple Objects, and Exceptions}

The result stated in Theorem 5 can be extended to cover some cases where, $E$ and $S$ exchange information not only through values of primitive types, but also arrays, simple object, and throwing exceptions. Some restrictions, however, have to be imposed on $I_{E}$ and the program $S$. These restrictions guarantee that, although references are exchanged between $E$ and $S$, the communication resembles exchange of pure data.

More precisely, our result works for the following class of systems $S$. Let $I_{E}$ be the minimal interface such that $I_{E} \vdash S$. We assume that:

1. Methods of classes in $I_{E}$ are static and their arguments are of primitive types or of type byte []. (Let us notice that this only restricts the way $S$ uses the environment; an environment that implements the interface $I_{E}$ can have arbitrary fields and methods. Note also that methods of $I_{E}$ can throw exceptions.)

2. Fields of classes in $I_{E}$ are non-static and of primitive types or of type byte[]. (Again, a system implementing $I_{E}$ is not restricted to such fields).

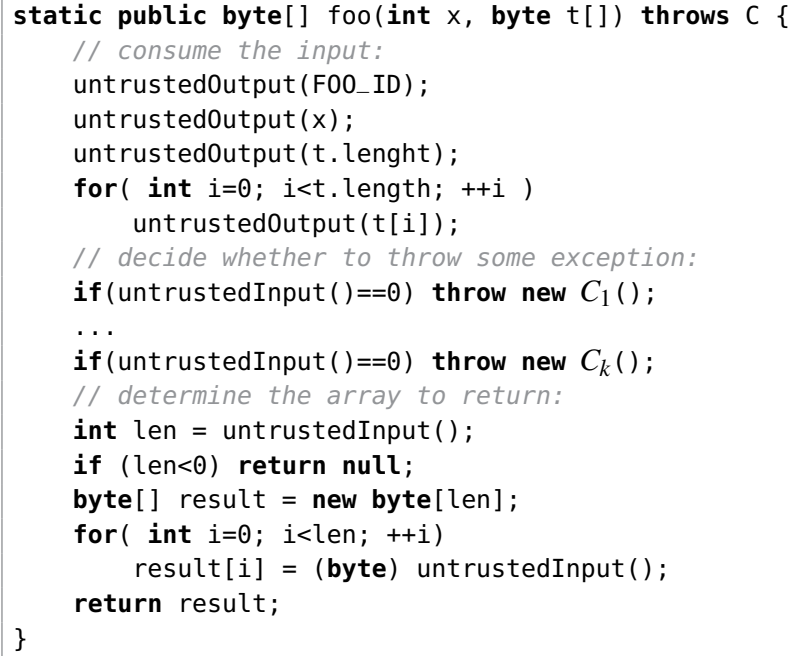

Figure 4. $\tilde{E}_{\vec{u}}$ : the implementation of a method with the signature static public byte[] foo(int $x$, byte[] t) throws $C$, where $C_{1}, \ldots, C_{k}$ are all subclasses of $\mathrm{c}$, including $\mathrm{c}$ itself, which are either standard exceptions or empty classes in $I_{E}$.

3. Whenever an array (i.e. the reference to an array) is passed to the environment, this reference is not used by $S$ afterwards. (This property can be easily guaranteed by a syntactical restriction to pass only fresh copies of arrays to the environment).

4. If $S$ calls a (static) method of $I_{E}$ which returns a reference $r$, the way $S$ can use $r$ is subject to the following restrictions: (1) If $r$ is a reference of type byte [ ], then $S$ is only allowed to immediately clone $r ; r$ is not used afterwards. (2) If $r$ is a reference to an object, then $S$ is only allowed to immediately clone the fields of $r$ (of primitive types and of type byte[]); $r$ is not used afterwards. $S$ cannot use $r$ in any way not specified explicitly above.

5. For every try-catch statement in $S$ of the form

$$
\operatorname{try}\{\ldots\} \operatorname{catch}(\mathrm{C} r)\{\mathrm{B}\}
$$

if $C$ or a subclass of $C$ is listed in the throws clause of some method in $I_{E}$ (and thus this statement may potentially catch an exception thrown by $E$ ), then $r$ is subject to the same restrictions as in item 4. case (2) above.

For such programs we can, as in the previous section, construct a fixed $\tilde{E}_{\vec{u}}$ such that it is enough to consider only these system (for all $\vec{u}$ ). This system consists of the static methods untrustedInput and untrustedoutput as defined above and, for every class $C$ of $I_{E}$, the declaration of $C$ with the implementation of (static) methods as illustrated by the example given in Figure 4. This example illustrates the case when an array is returned. When an object of class $D$ is to be returned, then a fresh object of this class is created and 
the values of its fields that are specified in $I_{E}$ are filled using untrustedInput, as for the array in the example.

The following theorem is a generalisation of Theorem 5 to the richer family of progarms considered in this section (see Appendix D-B for the proof).

Theorem 6. Let $S$ be as above. I-non-interference, for $I=\emptyset$, holds for $S$ if and only if for all sequences $\vec{u}$ as above noninterference holds for $\tilde{E}_{u} \cdot S$.

\section{PUBLIC-KEY ENCRYPTION}

In our case study, we will analyze a system that uses public-key encryption. We therefore now provide an ideal functionality for public-key encryption, denoted by IdealPKE. Our example program will be analyzed based on this functionality (see Section IX). Details of IdealPKE are given in Appendix A-A. We prove that this functionality can be realized by a system, which we call RealPKE, that implements, in the obvious way, an IND-CCA2-secure public-key encryption scheme.

The interface implemented by IdealPKE and RealPKE, denoted by $I_{P K E}$, consists of two classes:

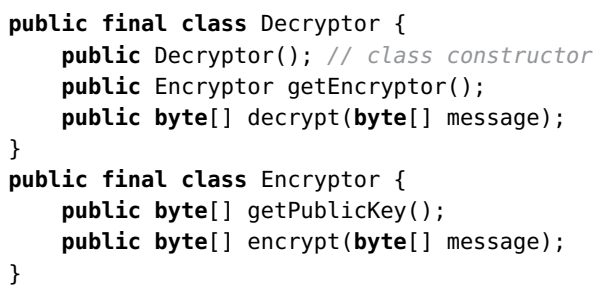

An object of class Decryptor is supposed to encapsulate a public/private key pair. These keys are created when the object is constructed. It allows a party who owns such an object to decrypt messages (for which, intuitively, the private key is needed) by the method decrypt. This party can also obtain an encryptor which encapsulates the related public key and encrypts messages via the method encrypt. The encapsulated public key can be obtained by the method getPublickey. Typically, the party who creates (owns) a decryptor gives away only an associated encryptor.

Our ideal functionality IdealPKE is in the spirit of ideal public-key functionalities in the cryptographic literature (see, e.g., [10], [27]). However, it is more restricted in that we do not (yet) model corruption in that functionality, and hence, in its realization. So far, corruption, if considered, needs to be modeled in the higher-level system using IdealPKE. In particular, whenever an encryptor object is used, it is guaranteed that the public key encapsulated in this object was honestly generated and no party has direct access to the corresponding private key.

While this might be too restricted and inconvenient in some scenarios (and it is interesting future work to extend this functionality), we took this design choice because our functionality facilitates the automated verification process and is nevertheless useful in interesting scenarios. We emphasize that the theorem for the realization of IdealPKE (Theorem 7) would hold for more expressive functionalities, in particular, those that model corruption and exactly resemble the ones that can be found in the cryptographic literature.

Our ideal functionality IdealPKE : $I_{P K E}$ is defined on top of the interface $I_{E n c}$ (which, in a complete system, is implemented by the environment or the simulator), i.e. $I_{E n c} \vdash$ IdealPKE, where $I_{E n c}$ is as follows:

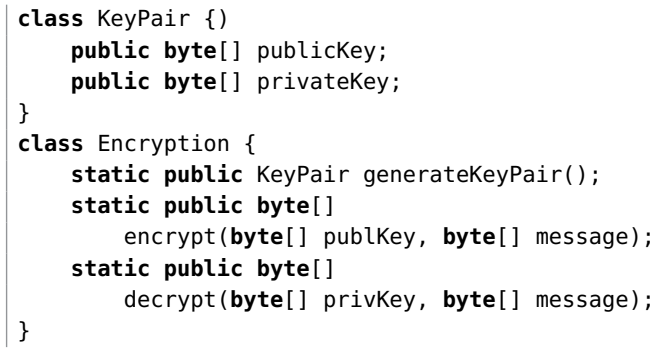

In a nutshell, IdealPKE works as follows: On initialization, via Decryptor ( ), a public/private key pair is created by calling generateKeyPair() in $I_{E n c}$. IdealPKE also maintains a list of message/ciphertext pairs; this list is shared with all associated encryptors (objects returned by getEncryptor). When method encrypt in $I_{P K E}$ is called for a message $m$, the ideal functionality calls encrypt in $I_{E n c}$ to encrypt a sequence of zeros of the same length, obtaining the ciphertext $m^{\prime}$, and stores $\left(m, m^{\prime}\right)$ in the list. The method decrypt in $I_{P K E}$ called for $m^{\prime}$ looks for a pair $\left(m, m^{\prime}\right)$ in the list and, if it finds it, returns $m$; otherwise, it decrypts $m^{\prime}$ (calling decrypt in $I_{E n c}$ ) obtaining $m^{\prime \prime}$ and returns this message. The idea behind this functionality is that the ciphertext returned by encryption is not related in any way (except for the length of the message) to the plaintext.

As already mentioned, RealPKE is defined in a straightforward way using any IND-CCA2-secure encryption scheme. The following theorem, which says that RealPKE in fact realizes IdealPKE, is proved in Appendix E.

Theorem 7. If RealPKE uses an IND-CCA2-secure encryption scheme, then RealPKE $\leq{ }^{\left(I_{P K E}, \emptyset, \emptyset, I_{E n c}\right)}$ IdealPKE.

\section{CASE STUdy}

In our case study and as a proof of concept of our framework, we consider a simple system that uses public-key encryption: clients send secrets encrypted over an untrusted network, controlled by an active adversary, to a server who decrypts the messages. This can be seen as a rudimentary way encryption can be used. Based on our framework, we use the Joana tool (see below), to verify strong secrecy of the messages sent over the network, i.e., non-interference is shown using Joana for the system when it runs with IdealPKE and by our framework we then obtain computational indistinguishability guarantees when IdealPKE is replaced by RealPKE. 
We emphasize that, while the code of client and server are quite small, the actual code that needs to be analyzed is larger because it includes the ideal functionality and the code that results from applying the techniques developed in Section VII-B (we note that the verified program is in the family of systems considered in this section); altogether the code in our case study comprises $376 \mathrm{LoC}$ in a rich fragment of Java. Moreover, the adversary model we consider in the case study is strong in that the (active) adversary dictates the number of clients, sends a pair of messages to every client of which one is encrypted (in the style of a left-right oracle), and controls the network.

The main goal of our case study is to demonstrate that it is, in principle, possible to establish strong cryptographic security guarantees for Java(-like) programs using existing tools for checking standard non-interference properties.

The verification of the program considered in our case study took just a few seconds (see below), and hence, our approach, in conjunction with Joana, should also work for bigger programs and more complex settings.

\section{A. The Analyzed Program}

We now describe the analyzed program in more detail (see Appendix A-B for the code).

Besides the code for the client and server, the program also contains a setup class which contains the methods main() and creates instances of protocol participants and organizes the communication. This setup first creates a public/private key pair (encapsulated in a decryptor object) for the server. In a while-loop it then expects, in every round, i) two input messages from the network (adversary), ii) depending on a static boolean variable secret (which will be declared to be high), one of the two messages is picked, iii) a client is created and it is given the public key of the server and the chosen message (the client will encrypt that message and send it over the network to the server), iv) a message from the network is expected, and v) given to the server, who will then decrypt this message and assign the plaintext to some variable.

We denote the class setup by $\operatorname{Setup}[b]$, where $b \in$ $\{$ false, true $\}$ is the value with which secret is initialized in Setup. By $S^{\text {real }}[b]$, for $b \in\{$ false, true $\}$, we denote the system consisting of the class Setup $[b]$, the class Client, the class Server, and the system RealPKE. This system is open: it uses unspecified network (and untrusted input from the environment) which is controlled by the adversary. Analogously, $S^{\text {ideal }}[b]$ contains IdealPKE instead of RealPKE. Note that $S^{\text {ideal }}[b]$ is even more open in that the ideal functionality asks the environment to encrypt and decrypt some messages (see the definition of IdealPKE).

We note that for the analysis with Joana we consider a variant of the ideal functionality where the ideal encryption is done always with the zero byte, and hence, the ideal functionality does not reveal the length of the encrypted message. (This is reasonable if only messages of fixed length are supposed to be encrypted.)

\section{B. The Property to be Proven}

The property we want to show is

$$
S^{\text {real }}[\text { false }] \approx_{\text {comp }}^{\emptyset} S^{\text {real }}[\text { true }],
$$

that is, the two variants of the system are indistinguishable from the point of view of an adversary who implements the networking, but does not call (directly) methods of $S^{\text {real }}[b]$; he, however, through the setup class, determines the number of clients that are created and the message pair for every client.

By our framework, to prove (4) it is enough to show $I$ non-interference of the system $S^{\text {ideal }}[b]$. Since the system $S^{\text {ideal }}[b]$ is in the class of systems considered in Section VII-B, we can use the results from that section which says that we only need to show (classical) non-interference of the system $T_{\vec{u}}[b]$, for all $\vec{u}$, which extends $S^{\text {ideal }}[b]$ by $\tilde{E}_{u}^{I_{E}}$, where $I_{E}$ is the interface IdealPKE extended with methods for network input and output.

To show that, for all $\vec{u}$, non-interference holds for $T_{\vec{u}}[b]$, we used the Joana tool, as described in the next section.

The verification carried out establishes (4), under the (reasonable) assumption that Joana is sound with respect to the subset of Java covered in Jinja+ (as explained in the next section, Joana has been proved to be sound with respect to the semantics of Jinja).

\section{Analysis with Joana}

Joana [20] is a static analysis tool. It uses a technique called slicing for program dependence graphs (PDG)-a graph-based representation of the program-to detect illegal information flows. It can handle full Java bytecode including exceptions. A machine-checked proof [37] provides a formal specification of PDGs and shows that slicing can be used to obtain a sound approximation of the information flows inside a program. Additional work [38], [39] verified that a variant of the slicing algorithm used by Joana can help to guarantee classical Goguen/Meseguer non-interference [16] (which is the kind of non-interference we are interested in) for the semantics of the Jinja language. The technique used by Joana does not depend on such details of the semantics as the maximum value of integers. Therefore, the guarantees Joana gives apply to all variants of the semantics that differ on this maximum value.

Joana is a whole-program analysis tool that analyzes an explicit version of a program and thus cannot reason about the security of a family of programs in general. We show, however, that this is possible for the specific families of (closed) programs $\tilde{E}_{\vec{u}} \cdot S$ (parametrized by $\vec{u}$ ), considered in Section VII-B (see Appendix F for details). In particular, Joana can verify (absence of) information flow for the family $T_{\vec{u}}$ defined in the previous section. We want to emphasize 
that the results of Appendix $F$ are not specific to the protocol analyzed in this case study; they enable Joana to reason about any system that complies with the restrictions of Section VII-B, and hence, these results are of general interest.

In the verification process we have carried out, we have marked the initialization of the variable secret as high input and modifications to the result variable of unstrusted0utput as low output. Then Joana automatically has built the PDG model of the program, marked the corresponding nodes in the graph with high and low, and checked if no information flow is possible from high input to low output through a data flow analysis on the graph. (In case an illegal flow is detected Joana issues a violation of the security property and returns the set of all possible paths (slice) of illegal flow in the program.)

Because of various precision enhancements in Joanaespecially detection of impossible NullPointerExceptions and object-sensitivity-we were able to analyze the given family of programs (without any false positives that might have stemmed from the involved overapproximations) and thus to guarantee the absence of information flow.

Joana took about 11 seconds on a standard PC to finish the analysis of the program (with a size of 376 LoC). PDG computation took 10 seconds and only 1 second was needed to detect the absence of illegal flow inside the PDG.

The complete source code that was analyzed can be download from [26].

\section{Related WORK}

As already mentioned in the introduction, our work contributes to the area of language-based analysis of software that uses cryptography, such as cryptographic protocols, an area that recently has gained much attention. We discuss some of the more closely related work in this area in what follows.

The work most closely related to our work is the work by Fournet et al. [14]. Fournet et al. also aim at establishing computational indistinguishability properties for systems written in a practical programming language; this work, in fact, seems to be the first to study such strong properties in the area of language-based cryptographic analysis, other work, including the work mentioned below, considers trace properties, such as authentication and weak secrecy. However, there are many differences between the work by Fournet et al. and our work, in terms of the results obtained, the approach taken, and the programming language considered. Fournet et al. consider a fragment of the functional language F\#, while we consider a fragment of Java. Their approach, with theorems of the form "if a program type-checks, then it has certain cryptographic properties", is strongly based on type checking (with refinement types), while the point of our framework is to enable different techniques and tools that a priori cannot deal with cryptography to establish cryptographic guarantees. While the results of Fournet et al. concern specific cryptographic primitives, we establish general results for ideal functionalities and their realizations. While simulation-based techniques are used in the proofs of the theorems in the work of Fournet et al., in our approach, ideal functionalities are part of the program to be analyzed by the tools; by our framework, the ideal functionalities can then be replaced by their realizations and for the resulting systems we obtain computational indistinguishability.

In most of the work on language-based analysis of cryptobased software the analysis is carried out based on a symbolic (Dolev-Yao), rather than a cryptographic model (see, e.g., [18], [6], [11], [8]). Some works obtain cryptographic guarantees by applying computational soundness results [5], [2], where the usual restrictions of computational soundness results apply [13], or by compiling the source code to a specification language that then can be analyzed by a specialized tool, namely CryptoVerif [9], for cryptographic analysis [7]. In contrast, our approach, just as the one by Fournet et al. discussed above, does not take a detour through symbolic models in combination with computational soundness results. Also, our approach does not rely on specialized tools for cryptographic analysis.

The existing works on the security analysis of cryptobased software has mostly focussed on fragments of F\# and $\mathrm{C}$, including the above mentioned works. Some works consider (fragments of) Java [22], [33], but in a symbolic model and without formal guarantees.

Our framework might also be applicable in the context of computational non-interference (see, e.g., [15], [28]) since computational non-interference can be seen as a specific form of computational indistinguishability. It is interesting future work to investigate the connection between these works and our work further.

Our work also contributes to the mostly unexplored field of non-interference for interactive/open systems [32], [12]. Our technique presented in Section VII enables program analysis tools for checking non-interference of closed systems to deal with open/interactive systems in a practical programming language, namely Java. Existing works on noninterference for interactive systems [32], [12] are orthogonal to our work in that on the one hand they consider abstract system models (labeled transition systems with input and output), rather than a practical programming language, and on the other hand they study systems w.r.t. a more general lattice of security labels for input/output channels.

Finally, we mention a line of work that considers methods for generating secure Java programs from abstract models of cryptographic protocols (see, e.g. [4]).

\section{CONCLUSION}

In this paper, we have presented a general framework for establishing computational indistinguishability properties for Java(-like) programs using program analysis tools that 
can check (standard) non-interference properties of Java programs but a priori cannot deal with cryptography and cryptographic adversaries, i.e., probabilistic polynomial-time adversaries. The approach we have proposed is new and combines techniques from program analysis and simulationbased security. Our framework is stated and proved for the Java-like language Jinja+, which comprises a rich fragment of Java.

As a proof of concept of our framework, we have used an automatic tool, namely Joana, to check the non-interference of a Java program. By our framework, this analysis implies computational indistinguishability for that program (w.r.t. an active adversary). The analysis performed by Joana was very fast and suggests that more complex systems can be analyzed within our framework using this tool. Our case study has thus demonstrated, for the first time, that general program analysis tools that a priori are not designed to perform cryptographic analysis of Java programs, can in fact be used for that purpose.

Our work contributes to the field of language-based analysis of crypto-based software, which recently has gained much attention, in that i) a new approach is proposed, ii) our approach works for a (rich fragment of a) practical programming language, namely Java, which has not gotten much attention so far in this area, and iii) computational indistinguishability guarantees are obtained, rather than only guarantees in more abstract symbolic (Dolev-Yao) models and rather than trace properties, such as authentication and weak secrecy, as in most other works, and iv) these guarantees are obtained directly without taking a detour through symbolic models in combination with computational soundness results, as in most other works.

There are many directions for future work. We briefly mention a few. First, we are confident that, besides Joana, also other program analysis tools can be used within our framework to establish cryptographic security properties of Java programs, with possible candidates being the interactive theorem prover $\mathrm{KeY}$ [1], a tool based on Maude [3], and Jif [29], [30].

Acknowledgment. This work was partially supported by Deutsche Forschungsgemeinschaft (DFG) under Grant KU 1434/6-1 within the priority programme 1496 "Reliably Secure Software Systems - RS ${ }^{3}$ ".

\section{REFERENCES}

[1] Wolfgang Ahrendt, Thomas Baar, Bernhard Beckert, Richard Bubel, Martin Giese, Reiner Hähnle, Wolfram Menzel, Wojciech Mostowski, Andreas Roth, Steffen Schlager, and Peter H. Schmitt. The KeY tool. Software and System Modeling, 4:32-54, 2005.

[2] Mihhail Aizatulin, Andrew D. Gordon, and Jan Jürjens. Extracting and verifying cryptographic models from $\mathrm{C}$ protocol code by symbolic execution. In Yan Chen, George Danezis, and Vitaly Shmatikov, editors, Proceedings of the 18th ACM
Conference on Computer and Communications Security (CCS 2011), pages 331-340. ACM, 2011.

[3] Mauricio Alba-Castro, María Alpuente, and Santiago Escobar. Abstract Certification of Global Non-interference in Rewriting Logic. In Frank S. de Boer, Marcello M. Bonsangue, Stefan Hallerstede, and Michael Leuschel, editors, Formal Methods for Components and Objects - 8th International Symposium (FMCO 2009). Revised Selected Papers, volume 6286 of Lecture Notes in Computer Science, pages 105-124. Springer, 2009.

[4] Matteo Avalle, Alfredo Pironti, Riccardo Sisto, and Davide Pozza. The JavaSPI framework for security protocol implementation. In Availability, Reliability and Security (ARES 11), page 746-751. IEEE Computer Society, IEEE Computer Society, 2011.

[5] Michael Backes, Matteo Maffei, and Dominique Unruh. Computationally sound verification of source code. In Ehab AlShaer, Angelos D. Keromytis, and Vitaly Shmatikov, editors, Proceedings of the 17th ACM Conference on Computer and Communications Security (CCS 2010), pages 387-398. ACM, 2010.

[6] K. Bhargavan, C. Fournet, A. D. Gordon, and S. Tse. Verified Interoperable Implementations of Security Protocols. In Proceedings of the 19th IEEE Computer Security Foundations Workshop (CSFW-19 2006), pages 139-152. IEEE Computer Society, 2006.

[7] Karthikeyan Bhargavan, Cédric Fournet, Ricardo Corin, and Eugen Zalinescu. Cryptographically verified implementations for TLS. In Peng Ning, Paul F. Syverson, and Somesh Jha, editors, Proceedings of the 2008 ACM Conference on Computer and Communications Security (CCS 2008), pages 459-468. ACM, 2008.

[8] Karthikeyan Bhargavan, Cédric Fournet, and Andrew D. Gordon. Modular verification of security protocol code by typing. In Manuel V. Hermenegildo and Jens Palsberg, editors, Proceedings of the 37th ACM SIGPLAN-SIGACT Symposium on Principles of Programming Languages (POPL 2010), pages 445-456. ACM, 2010.

[9] B. Blanchet. A Computationally Sound Mechanized Prover for Security Protocols. In IEEE Symposium on Security and Privacy (S\&P 2006), pages 140-154. IEEE Computer Society, 2006.

[10] R. Canetti. Universally Composable Security: A New Paradigm for Cryptographic Protocols. Technical Report 2000/067, Cryptology ePrint Archive, December 2005. http: //eprint.iacr.org/2000/067.

[11] S. Chaki and A. Datta. ASPIER: An automated framework for verifying security protocol implementations. In Proceedings of the 22nd IEEE Computer Security Foundations Symposium (CSF 2009), pages 172-185. IEEE Computer Society, 2009.

[12] David Clark and Sebastian Hunt. Non-Interference for Deterministic Interactive Programs. In Pierpaolo Degano, Joshua D. Guttman, and Fabio Martinelli, editors, Formal Aspects in Security and Trust, 5th International Workshop, FAST 2008, Revised Selected Papers, volume 5491 of Lecture Notes in Computer Science, pages 50-66. Springer, 2008. 
[13] Hubert Comon-Lundh and Véronique Cortier. How to prove security of communication protocols? A discussion on the soundness of formal models w.r.t. computational ones. In Thomas Schwentick and Christoph Dürr, editors, Proceedings of the 28th International Symposium on Theoretical Aspects of Computer Science (STACS 2011), volume 9 of LIPICs, pages 29-44. Schloss Dagstuhl - Leibniz-Zentrum fuer Informatik, 2011.

[14] Cédric Fournet, Markulf Kohlweiss, and Pierre-Yves Strub. Modular code-based cryptographic verification. In Yan Chen, George Danezis, and Vitaly Shmatikov, editors, Proceedings of the 18th ACM Conference on Computer and Communications Security (CCS 2011), pages 341-350. ACM, 2011.

[15] Cédric Fournet, Jérémy Planul, and Tamara Rezk. Information-flow types for homomorphic encryptions. In Yan Chen, George Danezis, and Vitaly Shmatikov, editors, Proceedings of the 18th ACM Conference on Computer and Communications Security (CCS 2011), pages 351-360. ACM, 2011.

[16] J. Goguen and J. Meseguer. Interference Control and Unwinding. In Proc. Symposium on Security and Privacy, pages 75-86. IEEE, 1984.

[17] Joseph A. Goguen and José Meseguer. Security Policies and Security Models. In Proceedings of IEEE Symposium on Security and Privacy, pages 11-20, 1982.

[18] J. Goubault-Larrecq and F. Parrennes. Cryptographic protocol analysis on real C code. In Verification, Model Checking, and Abstract Interpretation, 6th International Conference, VMCAI 2005, volume 5, pages 363-379. Springer, 2005.

[19] Jürgen Graf. Speeding up context-, object- and field-sensitive SDG generation. In 9th IEEE International Working Conference on Source Code Analysis and Manipulation, pages 105-114, September 2010.

[20] Christian Hammer and Gregor Snelting. Flow-Sensitive, Context-Sensitive, and Object-sensitive Information Flow Control Based on Program Dependence Graphs. International Journal of Information Security, 8(6):399-422, December 2009.

[21] Dennis Hofheinz, Dominique Unruh, and Jörn Müller-Quade. Polynomial Runtime and Composability. Technical Report 2009/023, Cryptology ePrint Archive, 2009. Available at http: //eprint.iacr.org/2009/023.

[22] Jan Jürjens. Security Analysis of Crypto-based Java Programs using Automated Theorem Provers. In 21st IEEE/ACM International Conference on Automated Software Engineering (ASE 2006), pages 167-176. IEEE Computer Society, 2006.

[23] Gerwin Klein and Tobias Nipkow. A Machine-Checked Model for a Java-Like Language, Virtual Machine, and Compiler. ACM Trans. Program. Lang. Syst., 28(4):619-695, 2006.

[24] R. Küsters. Simulation-Based Security with Inexhaustible Interactive Turing Machines. In Proceedings of the 19th IEEE Computer Security Foundations Workshop (CSFW-19 2006), pages 309-320. IEEE Computer Society, 2006.
[25] R. Küsters and M. Tuengerthal. Universally Composable Symmetric Encryption. In Proceedings of the 22nd IEEE Computer Security Foundations Symposium (CSF 2009), pages 293-307. IEEE Computer Society, 2009.

[26] Ralf Küsters, Tomasz Truderung, and Jürgen Graf. Source Code for Simple Protocol Case Study with Joana, 2012. Available at http://infsec.uni-trier.de/publications/software/ simplProtJoana.zip.

[27] Ralf Küsters and Max Tuengerthal. Joint State Theorems for Public-Key Encryption and Digitial Signature Functionalities with Local Computation. In Proceedings of the 21st IEEE Computer Security Foundations Symposium (CSF 2008), pages 270-284. IEEE Computer Society, 2008.

[28] Peeter Laud. Semantics and Program Analysis of Computationally Secure Information Flow. In David Sands, editor, Programming Languages and Systems, 10th European Symposium on Programming, ESOP 2001, volume 2028 of Lecture Notes in Computer Science, pages 77-91. Springer, 2001.

[29] A.C. Myers. JFlow: Practical mostly-static information flow control. In Proceedings of the 26th ACM SIGPLANSIGACT Symposium on Principles of Programming Languages (POPL), pages 228-241. ACM, 1999.

[30] Andrew C. Myers, Stephen Chong, Nathaniel Nystrom, Lantien Zheng, and Steve Zdancewic. Jif: Java Information Flow (software release), July 2001. http://www.cs.cornell. edu/jif/.

[31] Tobias Nipkow and David von Oheimb. Javalight is TypeSafe — Definitely. In POPL, pages 161-170, 1998

[32] Kevin R. O’Neill, Michael R. Clarkson, and Stephen Chong. Information-Flow Security for Interactive Programs. In 19th IEEE Computer Security Foundations Workshop (CSFW-19 2006), pages 190-201. IEEE Computer Society, 2006.

[33] Nicholas O'Shea. Using Elyjah to Analyse Java Implementations of Cryptographic Protocols. In Joint Workshop on Foundations of Computer Security, Automated Reasoning for Security Protocol Analysis and Issues in the Theory of Security (FCS-ARSPA-WITS-2008), 2008.

[34] Karl J. Ottenstein and Linda M. Ottenstein. The program dependence graph in a software development environment. In SDE 1: Proceedings of the first ACM SIGSOFT/SIGPLAN software engineering symposium on Practical software development environments, pages 177-184, New York, NY, USA, 1984. ACM.

[35] B. Pfitzmann and M. Waidner. A Model for Asynchronous Reactive Systems and its Application to Secure Message Transmission. In IEEE Symposium on Security and Privacy, pages 184-201. IEEE Computer Society Press, 2001.

[36] Andrei Sabelfeld and Andrew C. Myers. Language-Based Information-Flow Security. IEEE Journal on Selected Areas in Communications, special issue on Formal Methods for Security, 21(1):5-19, 2003. 
[37] Daniel Wasserrab. From Formal Semantics to Verified Slicing - A Modular Framework with Applications in Language Based Security. $\mathrm{PhD}$ thesis, Karlsruher Institut für Technologie, Fakultät für Informatik, October 2010.

[38] Daniel Wasserrab and Denis Lohner. Proving Information Flow Noninterference by Reusing a Machine-Checked Correctness Proof for Slicing. In 6th International Verification Workshop - VERIFY-2010, July 2010.

[39] Daniel Wasserrab, Denis Lohner, and Gregor Snelting. On PDG-Based Noninterference and its Modular Proof. In Proceedings of the 4th Workshop on Programming Languages and Analysis for Security, pages 31-44. ACM, June 2009.

[40] Mark Weiser. Program slicing. In Proceedings of the 5th international conference on Software engineering, ICSE '81, pages 439-449, Piscataway, NJ, USA, 1981. IEEE Press.

\section{APPENDIX A. PROGRAMS}

\section{A. Public Key Encryption}

Real Functionality. By RealPKE we denote the system consisting of the following classes and the system CCA2Enc.

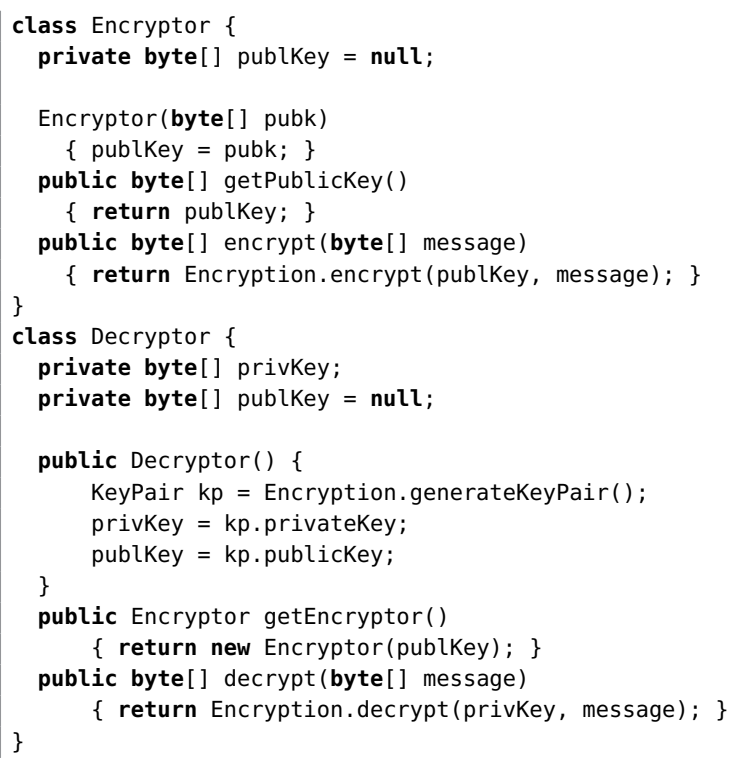

Ideal Functionality. By IdealPKE we denote the following system.

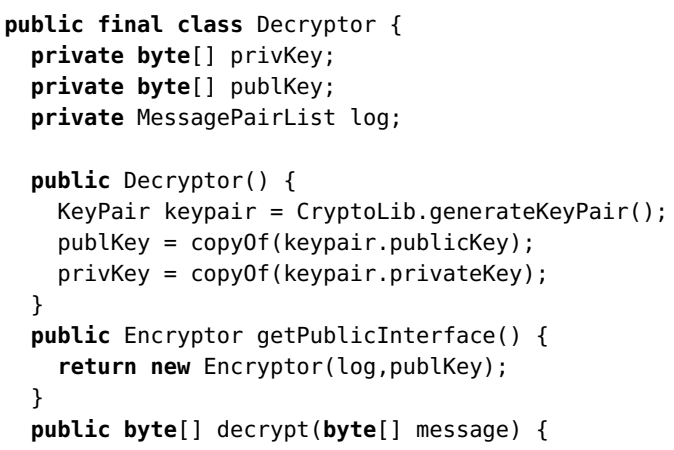

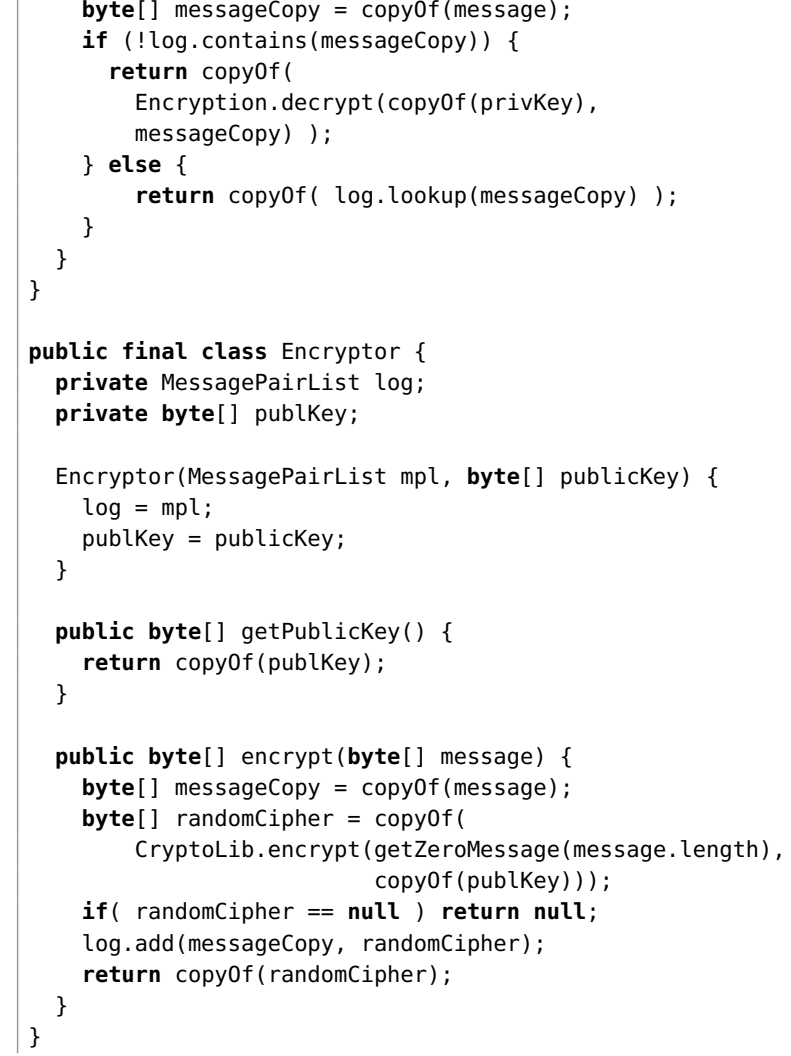

We omit here the declarations of (a) method getzeromessage that for a integer $n$ returns a message of length $n$ consisting of zeros, (b) method copyof that returns a copy of the given message, (c) class MessagePairlist that stores pairs of messages an offers natural methods: add (to add a message pair), contains (to check, for a message $m$, whether there is a pair $\left(m^{\prime}, m\right)$ in the list), and lookup (which, for a message $m$, returns $m^{\prime}$ as above, if it exists). The declaration of these methods can be found in [26].

\section{B. The Case Study}

We provide here complete code of the essential components (classes) of our case study: the client, the server, and the setup.

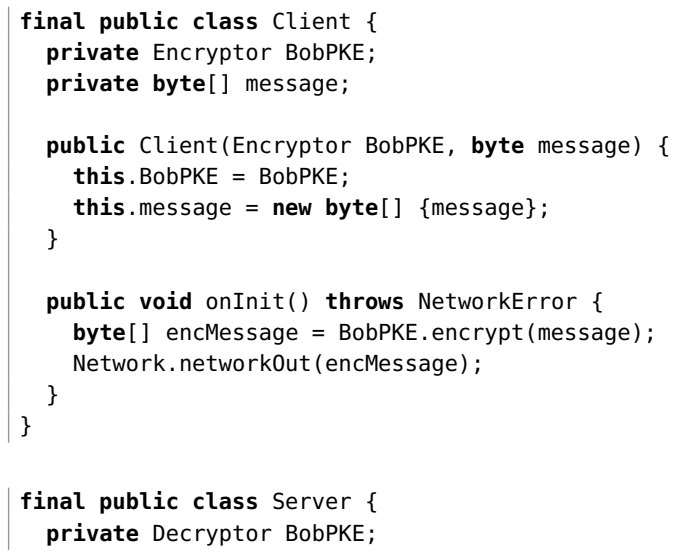




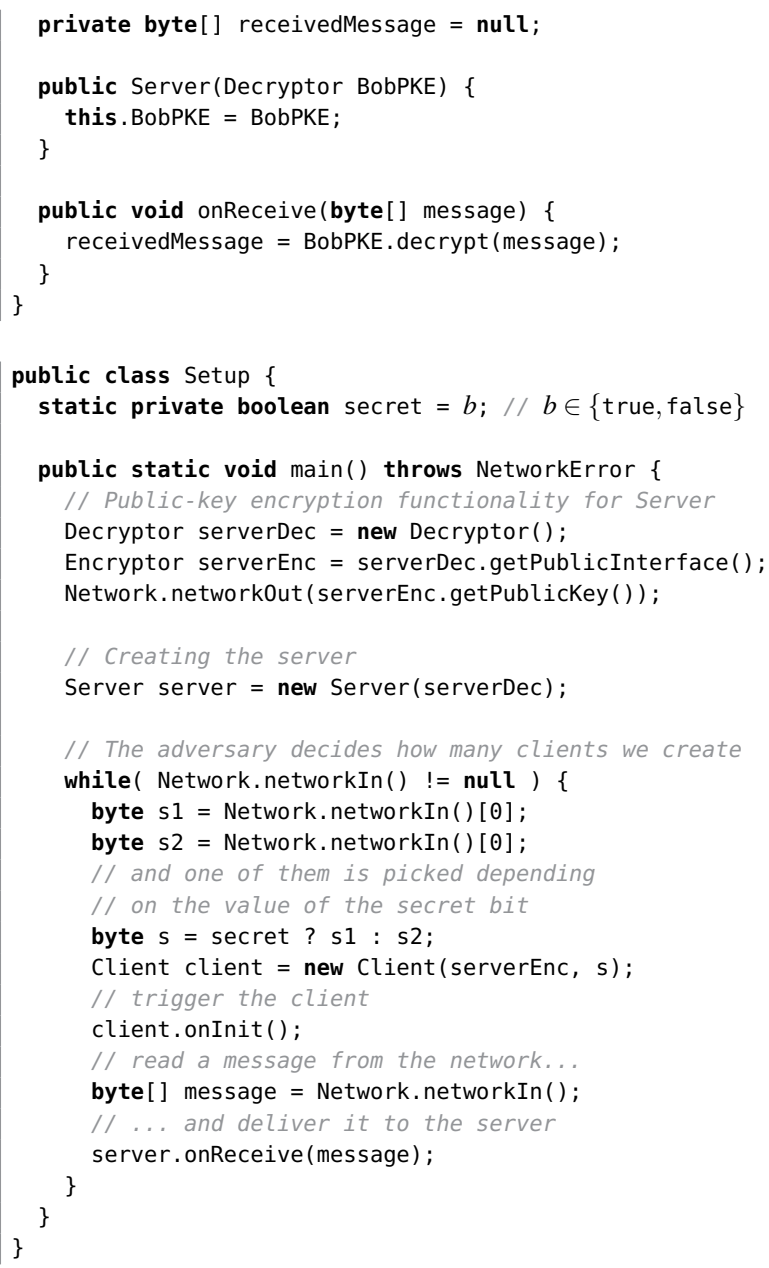

This program uses class Network (which we do not provide, as it is part of the environment) with the following interface:

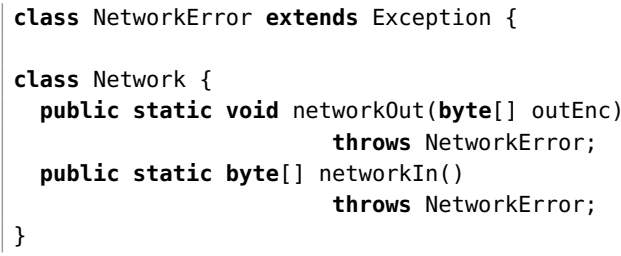

\section{APPENDIX B.} JINJA+

\section{A. Jinja+ Extensions}

As a basis of our formal results we take language Jinja+ that extends Jinja with: (a) the primitive type byte with natural conversions from and to int, (b) arrays, (c) abort primitive, (d) static fields (with the restriction that they can be initialized by literals only), (e) static methods, (f) access modifier for classes, fields, and methods (such as private, protected, and public), (g) final classes (classes that cannot be extended), (h) the throws clause of a method declaration (that declare which exceptions can be thrown by a method).
For the last three extensions-access modifiers, final classes, and throws clauses-we assume that they are provided by a compiler that, first, ensures that the policies expressed by access modifiers, the final modifier, and throws clauses are respected and then produces pure Jinja+ code (without access modifiers, the final modifier, and throws clauses). In the similar manner we can deal with constructors: a program using constructors can be easily translated to one without constructors (where creation and initialisation of an object is split into two separate steps).

The remaining extensions are described below:

Primitive types. The Jinja language, as specified in [23], offers only boolean and integer primitive types. For our purpose, we find it useful to also include type byte with natural conversions from and to int. Also, the set of operators on primitive types is extended to include the standard Java operators (such as multiplication). This extensions can be done in very straightforward way and, thus, we skip its detailed description.

Arrays. We will consider only one-dimensional arrays (an extension to multi-dimensional arrays is then quite straightforward; moreover multi-dimensional arrays can be simulated by nested arrays). To extend the Jinja language with one-dimensional arrays, we adopt the approach of [31].

First, we extend the set of types to include array types of the form $\tau_{\text {[] }}$, where $\tau$ is a type. Next, we extend the set of expressions by: (a) creation of new array: new $\tau[e]$, where $e$ is an expression (that is supposed to evaluate to an integer denoting the size of the array) and $\tau$ is a type, (b) array access: $e_{1}\left[e_{2}\right],(\mathrm{c})$ array length access: $e$.length, and (d) array assignment: $e_{1}\left[e_{2}\right]:=e_{3}$.

For this extension, following [31], we redefine a heap to be a map from references to objects, where an object is either an object instance, as defined above, or an array. An array is a triple consisting of its component type, its length $l$, and a table mapping $\{0, \ldots, l-1\}$ to values.

Extending (small-step) semantic rules to deal with arrays is quite straightforward (see the appendix).

The abort primitive. Expression abort, when evaluated, causes the program to stop. (Technically this expression cannot be reduced and causes the program execution to get stuck.)

Static methods and fields. Fields and methods can be declared as static. However, as can be seen below, to keep the semantics of the language simple, we impose some restrictions on initializers of static fields.

A static method does not require an object to be invoked. The syntax of static method call is C.f $(\operatorname{args})$, where C is the name of a class that provides $f$.

Extending Jinja with with static methods is straightforward. The rule for static method invocation is very similar to the one for non-static method invocation: the difference 
is that the variable this is not added to the context (block) within which the method body is executed (a static method cannot reference non-static fields and methods).

We assume that static fields can be initialized only with literals (constants) of appropriate types. If there is no explicit initializer, then a static variable is initialized with the default value of its type. For example, while static int $x=7$ and static int[] $t$ are valid declarations, the declaration static $A a=$ new $A()$ and static int $y=A$. foo() are not.

Dealing with more general static initializers is not difficult in principle, but it would require a precise-and quite complicated-model of the initialisation process, the complication we want to avoid.

Extending Jinja with static filed requires only a very little overhead: for a static field $f$ declared in class $c$ we introduce a global variable c.f (note that names of this form do not interfere with names of local variables and method parameters). These global variables are initialized before actual program (expression) is executed, as described in the definition of a run below.

Exceptions. A method declaration can contain a throws clause in which classes of exceptions that can be propagated by the method are listed. Such a clause can be omitted, in which case the above mentioned list is considered empty. When the meaning of throws clauses is considered, standard subtyping rules are applied (if class $A$ is listed in such a clause, then the method can propagate exceptions of class $A$ or any subclass of $A$ ).

As mentioned, we assume that the compiler (or a static verifier) statically checks whether the program complies with throws clauses.

Unlike in Java, however, we can assume without loss of generality that all exceptions must be declared in a throws clause if they are propagated by a method (in the Java terminology, we can say that all exceptions are checked). This will give us more control on the information which is passed between program components.

We consider the following hierarchy of standard (system) exceptions. In the root of this hierarchy we place (empty) class Exception. We require that only object of this class (and its subclasses) can be used as exceptions. Class SystemException, also empty, is a subclas of class Exception, and is a base class for the following system exceptions (exceptions which are not thrown explicitly, but may occur in result of some standard operations on expressions):

ArrayStoreException - trown to indicate an attempt to store an object of the wrong type into an array,

IndexOutOfBoundsException - thrown to indicate that an array has been indexed with an index being out of range,

NegativeArraySizeException - thrown to indicate an attempt to create an array with negative size,
NullPointerException - thrown if the null reference is used when an object is required,

ClassCastException - thrown to indicate an illegal cast.

We will assume that the above classes are predefined, and can be used in any program.

For completeness of the presentation, in this section we summarize all the rules of Jinjat. We start with rules of Jinja, following [23] (see this paper for the details on the used symbols). In particular, the syntactical convention used in these rules is that an application of a function $f$ to an argument $a$ is denoted by $f a$.

The rules assume a function binop that provides semantics for operations on atomic types. The exact definition of this function depends on the maximal size of integers that we consider (recall that we consider different variants of semantics for different size of integers given by intsize $(\eta)$ where $\eta$ is the security parameter).

\section{B. Rules of Jinja}

There are two point where our presentation rules diverge from the ones of [23]. First, as we assume unbounded memory, we do not have rules which throw 0utofMemoryError (and we assume that (new-Addr h) is never None). Second, we added labels to rules. These labels allow us to count the number of steps performed within (by) a given class or subsystem. A label $D$ in a step

$$
\langle e, s\rangle \stackrel{D}{\rightarrow}\left\langle e^{\prime}, s^{\prime}\right\rangle
$$

means, informally, that the step was executed by the code of class $D$. More precisely, the expression that was selected to be reduced by an elementary rule comes from a method of $D$. We use the label - if the origin of the reduced expression is not known (because, at that point, the context of this expression is not known; typically this empty label is overwritten by a subexpression reduction rule for blocks, that is rules (12)-(14)).

To define labeling of transitions, labels are also added to blocks that are obtained from the method call rule (a block is labeled by the name of the class from which the body of the method comes). Then, the labels of transitions are, roughly speaking, inherited from the innermost block within which the reduction takes place.

Now, for the run of a program $P$ with a subsystem $S$, we say that a step $\left\langle s_{1}, e_{1}\right\rangle \stackrel{D}{\rightarrow}\left\langle s_{2}, e_{2}\right\rangle$ is performed by $S$ and write $\left\langle s_{1}, e_{1}\right\rangle \stackrel{S}{\rightarrow}\left\langle s_{2}, e_{2}\right\rangle$, if $D$ is the name of a class defined in $S$.

Subexpression reduction rules (Figure 5) describe the order in which subexpressions are evaluated. The relation $[\rightarrow]$ it the extension of $\rightarrow$ to expression list $(\cdot$ is the list constructor).

Expression reduction rules (Figure 6) are applied when the subexpressions are sufficiently reduced. In the rule for 
method invocation, the required nested block structure is built with the help of the auciliary function blocks:

$$
\begin{gathered}
\operatorname{blocks}_{C}([],[],[], e)=e \\
\text { blocks }_{C}(V \cdot V s, T \cdot T s, v \cdot v s, e)= \\
=\{V: T ; V:=v ; \operatorname{blocks}(V s, T s, v s, e)\}_{C}
\end{gathered}
$$

(where - is the list constructor and [] denotes the empty list).

Exceptional reduction and exception propagation rules (Figure 7 and 8) describe how exception are thrown and propagated.

Note that we do not have a rule reducing abort. That means that, if this expression is to be reduced, the execution gets stuck.

\section{Rules for Jinja+}

In this section we present additional rules of Jinja+. Theres rules concern static method invocation and arrays. The rules are given in Figure 10 and 9.

\section{APPENDIX C. \\ PROOFS FOR SIMULATABILITY}

\section{A. Proof of Lemma 1}

Let $I, I_{E}, S_{1}, S_{2}$, and $E$ be given as stated in the lemma. We need to show that $E \cdot S_{2}$ is almost bounded and that $E \cdot S_{1} \equiv{ }_{\text {comp }} E \cdot S_{2}$.

Since $E \cdot S_{1}$ is almost bounded, there exists a polynomial $p$ such that the probability that the length of the run of this system with security parameter $\eta$ (and integer size intsize $(\eta)$ ) exceeds $p(\eta)$ is negligible. Now let us denote by $[E]$ the system that is defined just as $E$, but which in addition has a private static counter (defined in some new class in $E$ ) and where the code of $E$ is modified such that whenever a step in the code of $E$ is performed (according to the smallstep Jinja+ semantics), then the counter is increased. Once the bound $p(\eta)$ is reached, $[E]$ performs abort ().

By construction of $[E]$ it is easy to see that $[E]$ is a bounded environment because $[E]$ does not simulate more than $p(\eta)$ steps of $E$, where each step of $E$ can be simulated in a number of steps bounded by a constant. Also, $[E]$ behaves exactly like $E$ up to the point where the bound $p(\eta)$ is reached. From this, as further explained below, we obtain:

$$
E \cdot S_{1} \equiv_{\mathrm{comp}}[E] \cdot S_{1} \equiv_{\mathrm{comp}}[E] \cdot S_{2} \equiv_{\mathrm{comp}} E \cdot S_{2} .
$$

The first equivalence holds because $E$ reaches the bound $p(\eta)$ when running with $S_{1}$ only with negligible probability. Hence, the assignment of $E$ and $[E]$ to result is the same with overwhelming probability.

The second equivalence is true because $S_{1} \approx_{\text {comp }}^{I} S_{2}$ and $[E]$ is a bounded I-environment for $S_{1}$ and $S_{2}$.

The third equivalence holds because in the system $E \cdot S_{2}$ the bound $p(\eta)$ is reached also only with negligible probability: Otherwise, we could easily turn $[E]$ into a bounded environment $E^{\prime}$ that distinguishes $S_{1}$ and $S_{2}$, namely, $E^{\prime}$ works just as $[E]$ but outputs true, i.e., assigns true to the variable result iff the bound $p(\eta)$ is reached. So, if, when $E$ interacts with $S_{2}$, the bound were reached with nonnegligible probability, $E^{\prime}$ could distinguish between $S_{1}$ and $S_{2}$. It follows that $E \cdot S_{2}$ is almost bounded and that the last equivalence holds.

\section{B. Proof of Theorem 3}

Let $E$ be a bounded $I$-environment for $S_{1}$ (and hence, $S_{2}$ ). For a finite bit string $r$, let $E_{r}$ denote the deterministic system obtained from $E$ by fixing its randomness by $r$ in the following way: The primitive randomBit() is replaced by a method (along with a new static field) declared within $E_{r}$ such that the first $|r|$ bits returned by the method are chosen according to $r$; all the remaining bits returned by this method are 0 . It follows with $S_{1} \approx_{\text {perf }}^{I} S_{2}$ that (*) for all security parameters $\eta$, for all $r$, and for all integer sizes $s \geq 1$ such that $E_{r} \cdot S_{1}(\eta)$ and $E_{r} \cdot S_{2}(\eta)$ terminate for integer size $s$ it holds that $E_{r} \cdot S_{1}(\eta) \rightsquigarrow_{s}$ true iff $E_{r} \cdot S_{2}(\eta) \rightsquigarrow_{s}$ true. This implies

$$
E \cdot S_{1} \equiv_{\mathrm{comp}} E \cdot S_{2}
$$

because: By assumption, $E \cdot S_{1}$ and $E \cdot S_{2}$ are almost bounded. Hence, there exists a polynomial $p$ such that the probability that the length of a run (with integer size intsize $(\eta)$ ) of $E \cdot S_{1}(\eta)$ or $E \cdot S_{2}(\eta)$ exceeds $p(\eta)$ is negligible. So in all runs, except for a negligible fraction, at most $p(\eta)$ random bits are needed. Moreover, for almost all bit strings $r$ of length at most $p(\eta)$ and integers of size intsize $(\eta)$, we have that the runs of $E_{r} \cdot S_{1}(\eta)$ and $E_{r} \cdot S_{2}(\eta)$ terminate for integer size intsize $(\eta)$. Now by $(*)$ we know that for such $r$ the output of the runs of $E_{r} \cdot S_{1}(\eta)$ and $E_{r} \cdot S_{2}(\eta)$ with integer size intsize $(\eta)$ is the same.

Hence $S_{1} \approx_{\text {comp }}^{I} S_{2}$.

\section{APPENDIX D.}

\section{A Proof TECHNIQUe For Noninterference IN OPEN SYSTEMS}

\section{A. Proof of Theorem 5}

Let $I_{E}$ and $S$ be like in the theorem. Let $\emptyset \vdash E: I_{E}$ be an environment for $S$. We start with some definition that will be useful in the proof.

Below, we consider systems $E \cdot S$ such that the run of $E \cdot S$ is finite. We assume that $E$ and $S$ do not use the abort primitive (this assumptions simplifies some notation, but is not crucial; the proof without this assumption is similar).

Let $\rho$ be the run of the system $E \cdot S$. Let $(e, s)$ and $\left(e^{\prime}, s^{\prime}\right)$ be configurations in this run. We write $(e, s) \sim\left(e^{\prime}, s^{\prime}\right)$ if these configurations are equal up to references (addresses) remapping, i.e. if there exist a bijection $f$ from references to references that $(f(e), f(s))=\left(e^{\prime}, s^{\prime}\right)$, where $f(e)$ and $f(s)$ applies $f$ to every reference in $e$ and, respectively, in $s$. In the analogous way we define relation $s \sim s^{\prime}$ on states. 


$$
\begin{aligned}
& \frac{P \vdash\langle e, s\rangle \stackrel{\ell}{\rightarrow}\left\langle e^{\prime}, s^{\prime}\right\rangle}{P \vdash\langle\text { Cast } C e, s\rangle \stackrel{\ell}{\rightarrow}\left\langle\text { Cast } C e^{\prime}, s^{\prime}\right\rangle} \\
& \frac{P \vdash\langle e, s\rangle \stackrel{\ell}{\rightarrow}\left\langle e^{\prime}, s^{\prime}\right\rangle}{P \vdash\langle V:=e, s\rangle \stackrel{\ell}{\rightarrow}\left\langle V:=e^{\prime}, s^{\prime}\right\rangle} \\
& \frac{P \vdash\langle e, s\rangle \stackrel{\ell}{\rightarrow}\left\langle e^{\prime}, s^{\prime}\right\rangle}{P \vdash\langle e . F\{D\}, s\rangle \stackrel{\ell}{\rightarrow}\left\langle e^{\prime} . F\{D\}, s^{\prime}\right\rangle} \\
& \frac{P \vdash\langle e, s\rangle \stackrel{\ell}{\rightarrow}\left\langle e^{\prime}, s^{\prime}\right\rangle}{P \vdash\left\langle e . F\{D\}:=e_{2}, s\right\rangle \stackrel{\ell}{\rightarrow}\left\langle e^{\prime} . F\{D\}:=e_{2}, s^{\prime}\right\rangle} \\
& \frac{P \vdash\langle e, s\rangle \stackrel{\ell}{\rightarrow}\left\langle e^{\prime}, s^{\prime}\right\rangle}{P \vdash\langle\operatorname{Val} v \cdot F\{D\}:=e, s\rangle \stackrel{\ell}{\rightarrow}\left\langle\operatorname{Val} v \cdot F\{D\}:=e^{\prime}, s^{\prime}\right\rangle} \\
& \frac{P \vdash\langle e, s\rangle \stackrel{\ell}{\rightarrow}\left\langle e^{\prime}, s^{\prime}\right\rangle}{P \vdash\left\langle e \ll b o p \gg e_{2}, s\right\rangle \stackrel{\ell}{\rightarrow}\left\langle e^{\prime} \ll b o p \gg e_{2}, s^{\prime}\right\rangle} \\
& \frac{P \vdash\langle e, s\rangle \stackrel{\ell}{\rightarrow}\left\langle e^{\prime}, s^{\prime}\right\rangle}{P \vdash\left\langle\text { Val } v_{1} \ll b o p \gg e, s\right\rangle \stackrel{\ell}{\rightarrow}\left\langle\text { Val } v_{1} \ll b o p \gg e^{\prime}, s^{\prime}\right\rangle} \\
& \frac{P \vdash\langle e,(h, l(V:=\text { None }))\rangle \stackrel{\ell}{\rightarrow}\left\langle e^{\prime},\left(h^{\prime}, l^{\prime}\right)\right\rangle \quad l^{\prime} V=\text { None } \neg \text { assigned } V e}{P \vdash\left\langle\{V: T ; e\}_{D},(h, l)\right\rangle \stackrel{f(l, D)}{\rightarrow}\left\langle\left\{V: T ; e^{\prime}\right\}_{D},\left(h^{\prime}, l^{\prime}(V:=l V)\right)\right\rangle} \\
& \frac{P \vdash\langle e,(h, l(V:=\text { None }))\rangle \stackrel{\ell}{\rightarrow}\left\langle e^{\prime},\left(h^{\prime}, l^{\prime}\right)\right\rangle \quad l^{\prime} V=v \quad \neg \text { assigned } V e}{P \vdash\left\langle\{V: T ; e\}_{D},(h, l)\right\rangle \stackrel{f(l, D)}{\rightarrow}\left\langle\left\{V: T ; V:=\operatorname{Val} v ; e^{\prime}\right\}_{D},\left(h^{\prime}, l^{\prime}(V:=l V)\right)\right\rangle} \\
& \frac{P \vdash\langle e,(h, l(V \mapsto v))\rangle \stackrel{\ell}{\rightarrow}\left\langle e^{\prime},\left(h^{\prime}, l^{\prime}\right)\right\rangle \quad l^{\prime} V=v^{\prime}}{P \vdash\left\langle\{V: T ; V:=\operatorname{Val} v ; e\}_{D},(h, l)\right\rangle \stackrel{f(l, D)}{\longrightarrow}\left\langle\left\{V: T ; V:=\operatorname{Val} v^{\prime} ; e^{\prime}\right\}_{D},\left(h^{\prime}, l^{\prime}(V:=l V)\right)\right\rangle} \\
& \frac{P \vdash\langle e, s\rangle \stackrel{\ell}{\rightarrow}\left\langle e^{\prime}, s^{\prime}\right\rangle}{P \vdash\langle e . M(e s), s\rangle \stackrel{\ell}{\rightarrow}\left\langle e^{\prime} \cdot M(e s), s^{\prime}\right\rangle} \\
& \frac{P \vdash\langle e s, s\rangle[\stackrel{\ell}{\rightarrow}]\left\langle e s^{\prime}, s^{\prime}\right\rangle}{P \vdash\langle\operatorname{Val} v . M(e s), s\rangle \stackrel{\ell}{\rightarrow}\left\langle\operatorname{Val} v . M\left(e s^{\prime}\right), s^{\prime}\right\rangle} \\
& \frac{P \vdash\langle e, s\rangle \stackrel{\ell}{\rightarrow}\left\langle e^{\prime}, s^{\prime}\right\rangle}{P \vdash\left\langle e ; e_{2}, s\right\rangle \stackrel{\ell}{\rightarrow}\left\langle e^{\prime} ; e_{2}, s^{\prime}\right\rangle} \\
& \frac{P \vdash\langle e, s\rangle \stackrel{\ell}{\rightarrow}\left\langle e^{\prime}, s^{\prime}\right\rangle}{P \vdash\left\langle\text { if }(e) e_{1} \text { else } e_{2}, s\right\rangle \rightarrow\left\langle\text { if }\left(e^{\prime}\right) e_{1} \text { else } e_{2}, s^{\prime}\right\rangle} \\
& \frac{P \vdash\langle e, s\rangle \stackrel{\ell}{\rightarrow}\left\langle e^{\prime}, s^{\prime}\right\rangle}{P \vdash\langle e \cdot e s, s\rangle[\stackrel{\ell}{\rightarrow}]\left\langle e^{\prime} \cdot e s, s^{\prime}\right\rangle} \quad \frac{P \vdash\langle e s, s\rangle[\stackrel{\ell}{\rightarrow}]\left\langle e s^{\prime}, s^{\prime}\right\rangle}{P \vdash\langle\operatorname{Val} v \cdot e s, s\rangle[\stackrel{\ell}{\rightarrow}]\left\langle\operatorname{Val} v \cdot e s^{\prime}, s^{\prime}\right\rangle}
\end{aligned}
$$

Figure 5. Subexpression reduction rules. We define $f(l, D)=D$, if $l=-$; otherwise $f(l, D)=l$. 


$$
\begin{aligned}
& \text { new-Addr } h=a \quad P \vdash C \text { has-fields FDTs } \\
& P \vdash\langle\text { new } C,(h, l)\rangle \stackrel{-}{\longrightarrow}\langle\operatorname{addr} a,(h(a \mapsto(C, \text { init-fields } F D T s)), l)\rangle
\end{aligned}
$$

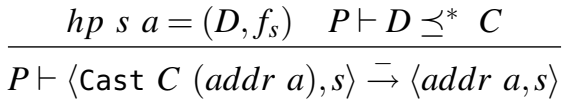

$$
\begin{aligned}
& P \vdash\langle\text { Cast } C \text { null }, s\rangle \stackrel{-}{\rightarrow} \text { null }, s\rangle \\
& \frac{l c l s V=v}{P \vdash\langle\operatorname{Var} V, s\rangle \rightarrow\langle\operatorname{Val} v, s\rangle} \\
& P \vdash\langle V:=\operatorname{Val} v,(h, l)\rangle \stackrel{-}{\rightarrow}\langle\text { unit },(h, l(V \mapsto v))\rangle \\
& \text { binop }\left(\text { bop }, v_{1}, v_{2}\right)=v \\
& \overline{P \vdash\left\langle\text { Val } v_{1} \ll \text { bop } \gg \operatorname{Val} v_{2}, s\right\rangle \stackrel{-}{\longrightarrow}\langle\text { Val } v, s\rangle} \\
& \frac{h p s a=(C, f s) \quad f s(F, D)=v}{P \vdash\langle\text { addr a.F }\{D\}, s\rangle \stackrel{-}{\rightarrow}\langle\text { Val } v, s\rangle} \\
& \frac{h a=(C, f s)}{P \vdash\langle\text { addr a.F }\{D\}:=\operatorname{Val} v,(h, l)\rangle \stackrel{-}{\rightarrow}\langle\text { unit },(h(a \mapsto(C, f s((F, D) \mapsto v))), l)\rangle} \\
& \underline{h p} s a=(C, f s) \quad P \vdash C \text { sees } M: T s \rightarrow T=(p n s, \text { body }) \text { in } D \quad|v s|=|p n s| \quad|T s|=|p n s|
\end{aligned}
$$

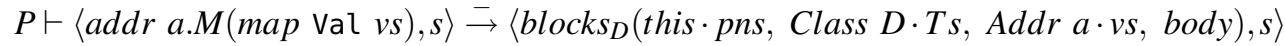

$$
\begin{aligned}
& P \vdash\left\langle\{V: T ; V:=\operatorname{Val} v ; \operatorname{Val} u\}_{D}, s\right\rangle \stackrel{D}{\rightarrow}\langle\operatorname{Val} u, s\rangle \\
& P \vdash\langle\{V: T ; \operatorname{Val} u\}, s\rangle \stackrel{-}{\rightarrow}\langle\operatorname{Val} u, s\rangle \\
& P \vdash\left\langle\operatorname{Val} v ; e_{2}, s\right\rangle \stackrel{-}{\longrightarrow}\left\langle e_{2}, s\right\rangle \\
& P \vdash\left\langle\text { if }(\text { true }) e_{1} \text { else } e_{2}, s\right\rangle \rightarrow\left\langle e_{1}, s\right\rangle \\
& P \vdash\left\langle\text { if }(\text { false }) e_{1} \text { else } e_{2}, s\right\rangle \stackrel{-}{\longrightarrow}\left\langle e_{2}, s\right\rangle \\
& P \vdash\langle\text { while }(b) c, s\rangle \stackrel{-}{\rightarrow}\langle\operatorname{if}(b)(c \text {; while }(b) c) \text { else unit, } s\rangle
\end{aligned}
$$

Figure 6. Expression reduction

Let $s=(h, l)$ be a state. By $\left.s\right|_{E}=\left(\left.h\right|_{E},\left.l\right|_{E}\right)$ we denote the part of the state that is accessible from $E$ through the static variables that $E$ uses. Formally, we leave in the domain of $\left.l\right|_{E}$ only those static variables of $l$ that $E$ can access; we leave in the domain of $\left.h\right|_{E}$ only those references that can be reached from those static variables, where a reference can be reached from $\left.l\right|_{E}$ if (i) it is stored in one of the variables of $\left.l\right|_{E}$ or (ii) it is stored in an object that can be reached from $\left.l\right|_{E}$.

In the analogous way we define $\left.s\right|_{S}$.
We can split the run $\rho$ into segments

$$
A_{1}, B_{1}, A_{2} \ldots, B_{k-1}, A_{k}
$$

such that:

- Every $A_{i}$ is a sequence of states (sub-run) where code of $S$ is executed (formally transitions within $A_{k}$ are labeled by names of classes from $E$ ). Moreover, every $A_{i}$ except for the last one ends with a state of the form $\left(e_{i}\left[C_{i} \cdot m_{i}\left(\vec{a}_{i}\right)\right], s_{i}\right)$ where the subexpression $C_{i} \cdot m_{i}\left(\vec{a}_{i}\right)$ is about to be rewritten (with $C_{i}$ defined in $E$ ). We will denote the tuple $\left(C_{i}, m_{i}, \vec{a}_{i}\right)$ 


$$
\begin{aligned}
& \text { hp s } a=(D, f s) \quad \neg P \vdash D \preceq^{*} C \\
& P \vdash\langle\text { Cast } C(\operatorname{addr} a), s\rangle \stackrel{-}{\longrightarrow}\langle\text { THROW ClassCastException, } s\rangle \\
& P \vdash\langle\text { null.F }\{D\}, s\rangle \stackrel{-}{\rightarrow} \text { THROW NullPointerException, } s\rangle
\end{aligned}
$$

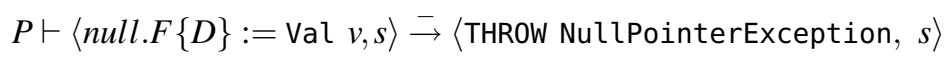

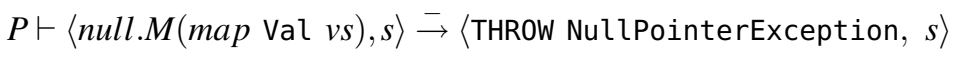

$$
\begin{aligned}
& \frac{P \vdash\langle e, s\rangle \stackrel{\ell}{\rightarrow}\left\langle e^{\prime}, s^{\prime}\right\rangle}{P \vdash\langle\text { throw } e, s\rangle \stackrel{\ell}{\rightarrow}\left\langle\text { throw } e^{\prime}, s^{\prime}\right\rangle} \\
& P \vdash\langle\text { throw null, } s\rangle \stackrel{-}{\rightarrow}\langle\text { THROW NullPointerException, } s\rangle \\
& \frac{P \vdash\langle e, s\rangle \stackrel{\ell}{\rightarrow}\left\langle e^{\prime}, s^{\prime}\right\rangle}{P \vdash\left\langle\operatorname{try} e \operatorname{catch}(C V) e_{2}, s\right\rangle \stackrel{\ell}{\rightarrow}\left\langle\operatorname{try} e^{\prime} \operatorname{catch}(C V) e_{2}, s^{\prime}\right\rangle} \\
& P \vdash\left\langle\operatorname{try} \operatorname{Val} v \text { catch }(C V) e_{2}, s\right\rangle \stackrel{-}{\rightarrow}\langle\operatorname{Val} v, s\rangle \\
& \frac{h p \text { s } a=(D, f s) \quad P \vdash D \preceq^{*} C}{P \vdash\left\langle\text { try THROW } a \text { catch }(C V) e_{2}, s\right\rangle \stackrel{-}{\rightarrow}\left\langle\left\{V: \text { Class } C ; V:=\operatorname{addr} a ; e_{2}\right\}, s\right\rangle} \\
& \frac{h p s a=(D, f s) \neg P \vdash D \preceq^{*} C}{P \vdash\left\langle\text { try THROW } a \text { catch }(C V) e_{2}, s\right\rangle \stackrel{-}{\longrightarrow}\langle\text { Throw } a, s\rangle}
\end{aligned}
$$

Figure 7. Exceptional expression reduction

by $x_{i}$.

- Every $B_{i}$ is a sequence of states where code of $E$ is executed. It begins with $\left(e_{i}\left[\left\{e_{i}^{\prime}\right\}_{C_{i}}\right], s_{i}\right)$, where $\left\{e_{i}^{\prime}\right\}_{C_{i}}$ is the block obtained by the static method call rule applied to $C_{i} \cdot m_{i}\left(\vec{a}_{i}\right)$ (it depends only on $C_{i}, m_{i}$, and $\vec{a}_{i}$ ), and ends with $\left(e_{i}\left[\left\{y_{i}\right\}_{C_{i}}\right], t_{i}\right)$, where $y_{i}$ is a value (that is return by this method).

We will represent such a run as

$$
\rho=A_{1}\left[s_{1}, x_{1}\right] B_{1}\left[t_{1}, y_{1}\right] A_{2}\left[s_{2}, x_{2}\right] \ldots B_{k-1}\left[t_{k-1}, y_{k-1}\right] A_{k}
$$

The square brackets, intuitively, contain all the information that is passed between $S$ and $E$. We will write $A_{i}^{\rho}$ to denote $A_{i}$, and similarly for $A_{i}, B_{i}, x_{i}, y_{i}, s_{i}$, and $t_{i}$ when it is necessary to make it explicit which run we consider.

The following result states, so called, state separation of $E$ and $S$ : for a representation of a run as above, $B_{i}$ does not change the part of the state that can be reached from $S$ and, similarly, $A_{i}$ does not change the part of the state that can be reached by $E$.

Lemma 4. We have:

1) $\left.s_{i}\right|_{S}=\left.t_{i}\right|_{S}$,

2) $\left.t_{i}\right|_{E}=\left.s_{i+1}\right|_{E}$.
Intuitively, this lemma holds true, because $E$ and $S$ do not exchange references (they exchange only primitive values) and do not share any static variables.

Because of this state separation, we can obtain the following two results. The first one states that the state of $E$ (the part of the state that $E$ can access) and the values that $E$ returns depend solely on the input it explicitly gets from $S$ by method calls (recall that the values passed by such calls are, by our assumption, of primitive types only).

Lemma 5. Let $S, S^{\prime}$ and $E$ be like in the theorem. Let $\rho$ be the run of $E \cdot S$ and $\rho^{\prime}$ be the run of $E \cdot S^{\prime}$. If

$$
x_{1}^{\rho}=x_{1}^{\rho^{\prime}}, \ldots, x_{k}^{\rho}=x_{k}^{\rho^{\prime}},
$$

then

$$
\left.\left.t_{k}^{\rho}\right|_{E} \sim t_{k}^{\rho^{\prime}}\right|_{E} \quad \text { and } \quad y_{k}^{\rho}=y_{k}^{\rho^{\prime}} .
$$

Conversely, the state of $S$ and the values it provides to $E$, solely depend on the values that $E$ returns to $S$ :

Lemma 6. Let $S, E$ and $E^{\prime}$ be like in the theorem. Let $\rho$ be the run of $E \cdot S$ and $\rho^{\prime}$ be the run of $E^{\prime} \cdot S$. If

$$
y_{1}^{\rho}=y_{1}^{\rho^{\prime}}, \ldots, y_{k}^{\rho}=y_{k}^{\rho^{\prime}},
$$




$$
\begin{aligned}
& P \vdash\langle\text { Cast } C \text { (throw } e), s\rangle \stackrel{-}{\longrightarrow}\langle\text { throw } e, s\rangle \\
& P \vdash\langle V:=\text { throw } e, s\rangle \stackrel{-}{\rightarrow} \text { throw } e, s\rangle \\
& P \vdash\langle\text { throw } e . F\{D\}, s\rangle \stackrel{-}{\rightarrow} \text { throw } e, s\rangle \\
& P \vdash\left\langle\text { throw } e . F\{D\}:=e_{2}, s\right\rangle \rightarrow\langle\text { throw } e, s\rangle \\
& P \vdash\langle\text { Val v.F }\{D\}:=\text { throw } e, s\rangle \stackrel{-}{\rightarrow}\langle\text { throw } e, s\rangle \\
& P \vdash\left\langle\text { throw } e \ll \text { bop } \gg e_{2}, s\right\rangle \stackrel{-}{\rightarrow}\langle\text { throw } e, s\rangle \\
& P \vdash\left\langle\operatorname{Val} v_{1} \ll \text { bop } \gg \text { throw } e, s\right\rangle \stackrel{-}{\rightarrow}\langle\text { throw } e, s\rangle \\
& P \vdash\left\langle\{V: T ; \text { Throw } a\}_{D}, s\right\rangle \stackrel{D}{\rightarrow}\langle\text { Throw } a, s\rangle \\
& P \vdash\left\langle\{V: T ; V:=\operatorname{Val} v ; \text { Throw } a\}_{D}, s\right\rangle \stackrel{D}{\rightarrow}\langle\text { Throw } a, s\rangle \\
& P \vdash\langle\text { throw } e . M(e s), s\rangle \stackrel{-}{\rightarrow}\langle\text { throw } e, s\rangle \\
& \left.P \vdash\left\langle\operatorname{Val} v . M\left(\text { map Val vs @ (throw } e \cdot e s^{\prime}\right)\right), s\right\rangle \stackrel{-}{\longrightarrow}\langle\text { throw } e, s\rangle \\
& \left.P \vdash\left\langle\text { throw } e ; e_{2}, s\right\rangle \stackrel{-}{\longrightarrow} \text { throw } e, s\right\rangle \\
& P \vdash\left\langle\text { if }(\text { throw } e) e_{1} \text { else } e_{2}, s\right\rangle \stackrel{-}{\rightarrow}\langle\text { throw } e, s\rangle \\
& P \vdash\langle\text { throw }(\text { throw } e), s\rangle \stackrel{-}{\rightarrow}\langle\text { throw } e, s\rangle \\
& \frac{P \vdash\langle e s, s\rangle[\stackrel{\ell}{\rightarrow}]\left\langle e s^{\prime}, s^{\prime}\right\rangle}{P \vdash\langle D \cdot M(e s), s\rangle \stackrel{\ell}{\rightarrow}\left\langle D \cdot M\left(e s^{\prime}\right), s^{\prime}\right\rangle} \\
& \frac{P \vdash\langle e, s\rangle \stackrel{\ell}{\rightarrow}\left\langle e^{\prime}, s^{\prime}\right\rangle}{P \vdash\left\langle e\left[e_{2}\right], s\right\rangle \stackrel{\ell}{\rightarrow}\left\langle e^{\prime}\left[e_{2}\right], s^{\prime}\right\rangle} \\
& \frac{P \vdash\langle e, s\rangle \stackrel{\ell}{\rightarrow}\left\langle e^{\prime}, s^{\prime}\right\rangle}{P \vdash\langle(\operatorname{Val} v)[e], s\rangle \stackrel{\ell}{\rightarrow}\left\langle(\operatorname{Val} v)\left[e^{\prime}\right], s^{\prime}\right\rangle} \\
& \left.P \vdash\left\langle D . M\left(\text { map Val vs @ (throw } e \cdot e s^{\prime}\right)\right), s\right\rangle \rightarrow\langle\text { throw } e, s\rangle \\
& P \vdash\left\langle(\text { throw } e)\left[e^{\prime}\right], s\right\rangle \stackrel{-}{\longrightarrow}\langle\text { throw } e, s\rangle \\
& \left.P \vdash\left\langle e^{\prime}[\text { throw } e], s\right\rangle \stackrel{-}{\rightarrow} \text { throw } e, s\right\rangle
\end{aligned}
$$

Figure 9. Subexpression reduction and exception propagation rules for Jinja+. 


$$
\begin{aligned}
& \frac{P \vdash D \text { has-static } M: T s \rightarrow T=(\text { pns, body }) \quad|v s|=|p n s| \quad|T s|=|p n s|}{P \vdash\langle D . M(\text { map } \operatorname{Val} v s), s\rangle \stackrel{-}{\rightarrow}\left\langle\text { blocks }_{D}(\text { pns }, T s, v s, \text { body }), s\right\rangle} \\
& \frac{n \geq 0, \text { new-Addr } h=a}{P \vdash\langle\text { new } \tau[\operatorname{intg}(n)],(h, l)\rangle \rightarrow\langle\text { addr } a,(h(a \mapsto \operatorname{initArr}(\tau, n)), l)\rangle} \\
& P \vdash\langle\text { null.F }\{D\}, s\rangle \stackrel{-}{\rightarrow} \text { THROW NullPointerException, } s\rangle \\
& n<0 \\
& \overline{P \vdash\langle\text { new } \tau[\operatorname{intg}(n)],(h, l)\rangle \rightarrow\langle\text { THROW NegativeArraySizeException, }(h, l)\rangle} \\
& \frac{h a=(\tau, m, t), 0 \leq n<m, t(n)=v}{P \vdash\langle(\text { addr } a) \text { [intg } n],(h, l)\rangle \rightarrow\langle\text { Val } v,(h, l)\rangle} \\
& \frac{h a=(\tau, m, t), \neg(0 \leq n<m),}{P \vdash\langle(\text { addr } a) \text { [intg } n],(h, l)\rangle \rightarrow\langle\text { THROW Index0ut0fBoundsException, }(h, l)\rangle} \\
& \frac{h a=(\tau, m, t),}{P \vdash\langle(\text { addr } a) . \text { lenght },(h, l)\rangle \rightarrow\langle\text { intg } m,(h, l)\rangle} \\
& \frac{h a=(\tau, m, t), 0 \leq n<m, \text { isOfType }(v, \tau), t^{\prime}=\operatorname{arrayUpdate}(t, n, v)}{P \vdash\left\langle(\text { addr a) }[\text { intg } n]:=\operatorname{Val} v,(h, l)\rangle \rightarrow\left\langle\text { Unit },\left(h\left(a \mapsto\left(\tau, m, t^{\prime}\right)\right), l\right)\right\rangle\right.} \\
& h a=(\tau, m, t), \neg(0 \leq n<m), \\
& \overline{P \vdash\langle(\text { addr } a)[\text { intg } n]}:=\operatorname{Val} v,(h, l)\rangle \rightarrow\langle\text { THROW IndexOut0fBoundsException, }(h, l)\rangle \\
& h a=(\tau, m, t), 0 \leq n<m, \neg \text { isOfType }(v, \tau), \\
& \overline{P \vdash\langle(\text { addr } a)[\operatorname{intg} n]:=\operatorname{Val} v,(h, l)\rangle \rightarrow\langle\text { THROW ArrayStoreException, }(h, l)\rangle}
\end{aligned}
$$

Figure 10. (Exceptional) expression reduction rules for Jinja+, where: Function initArr $(\tau, n)$ returns an array of length $n$ with elements initialized to the default value of type $\tau$. Expression $P \vdash D$ has-static $M: T s \rightarrow T=(p b s, b o d y)$ means that in program $P$, class $D$ contains declaration of static method $M$ with argument types $T s$, return type $T$, formal arguments $p b s$, and the body body.

then

$$
s_{k+1}^{\rho}\left|S \sim s_{k+1}^{\rho^{\prime}}\right| S \quad \text { and } \quad x_{k+1}^{\rho}=x_{k+1}^{\rho^{\prime}}
$$

Proof of Theorem 5. Implication from left to right is obvious. So let us assume that, $I$-non-interference does not hold for $S$. It means that there exists an $I$-environment $E$ for $S$ such that non-interference does not hold for $E \cdot S$. This, in turn, means that there are valid $\vec{a}_{1}$ and $\vec{a}_{2}$ such that the run $\rho$ of $E \cdot S\left[a_{1}\right]$ and the run $\rho^{\prime}$ of $E \cdot S\left[a_{1}\right]$ give different results (i.e. both runs are finite and the final value of result is different).

In the following, we only consider the case where the number of blocks $B_{i}$ in both runs is the same (the other case can be handled in a similar way).

As the value of result in a state $s$ is part of $\left.s\right|_{E}$, we conclude from Lemma 5, that there exists an index $k$ such that $x_{k}^{\rho} \neq x_{k}^{\rho^{\prime}}$. Let $k$ be the first such index. Note that $y_{i}^{\rho}=y_{i}^{\rho^{\prime}}$ for $i \in\{1, \ldots, k-1\}$. Let us assume that the first argument in the call described by $x_{k}^{\rho}$ has value $z$ which is different than the value $z^{\prime}$ of the first argument in $x_{k}^{\rho^{\prime}}$ (for the other cases the proof is very similar).

We define now a sequence $\vec{u}$ as the sequence containing only zeros with the following exceptions:

- In the system $\tilde{E}_{\vec{u}} \cdot S\left[a_{i}\right]$, the consecutive $(k-1)$ values that methods of $\tilde{E}_{\vec{u}}$ return to $S\left[a_{i}\right]$ are determined by some subsequence $u_{p_{1}}, \ldots, u_{p_{k}}$. Therefore we set $u_{p_{i}}=y_{i}$ for $i \in\{1, \ldots, k-1\}$ (that is, the values returned in $\tilde{E}_{\vec{u}} \cdot S\left[a_{i}\right]$ coincide with the values returned in $E \cdot S\left[a_{i}\right]$ ).

- Let $l$ be the integer such that $u_{l}$ decides whether to test the first arguments in $B_{k}$-th block (then this argument is compared to $u_{l+1}$ to determine the result). We set $u_{l}$ to 1 and $u_{l+1}$ to $z$ (as defined above). Note that $l \notin\left\{p_{1}, \ldots, p_{k-1}\right\}$.

- As mentioned, for all remaining $i$ we set $u_{i}=0$.

Now, to complete the proof it is enough to show that $\tilde{E}_{\vec{u}} \cdot S\left[a_{1}\right]$ and $\tilde{E}_{\vec{u}} \cdot S\left[a_{2}\right]$ give different results.

Let $\sigma$ be the run of the system $\tilde{E}_{\vec{u}} \cdot S\left[a_{1}\right]$ and $\sigma^{\prime}$ be the run of the system $\tilde{E}_{\vec{u}} \cdot S\left[a_{2}\right]$. As, by the definition of $\vec{u}$, we know that $y_{i}^{\rho}=y_{i}^{\rho^{\prime}}=y_{i}^{\sigma}=y_{i}^{\sigma^{\prime}}$ for $i \in\{1, \ldots, k-1\}$, we can use Lemma 6 to obtain $x_{k}^{\sigma}=x_{k}^{\rho}$ and $x_{k}^{\sigma^{\prime}}=x_{k}^{\rho^{\prime}}$. Therefore, the first 
argument in $x_{k}^{\sigma}$ is $z$ and it is different that the first argument in $x_{k}^{\sigma^{\prime}}$. Therefore, by the definition of $\vec{u}$ (more precisely, by the values of $u_{l}$ and $u_{l+1}$ the variable result is set to true in $\tilde{E}_{\vec{u}} \cdot S\left[a_{1}\right]$ and to false in $\tilde{E}_{\vec{u}} \cdot S\left[a_{2}\right]$, after which both systems terminate (the abort is executed immediately after the assignment). Hence, these systems give different results, which completes the proof.

\section{B. Communication through Arrays, Simple Objects, and Exceptions}

The proof of Theorem 6 is very similar-and only slightly more complicated-to the proof of Theorem 5. Indeed, the assumptions we have taken about the program $S$ guarantee that-even if, technically, some reference are exchanged between $E$ and $S$-the communication between $E$ and $S$ is, effectively, as if only pure values were exchanged.

Because of that we only mention those points in the proof which differ from the analogous points in the proof of Theorem 5.

1. There are the following changes in the representation of a run: (a) $x_{i}$ now records not only primitive values of arguments, but also values of arrays of bytes (not the references to these arrays though); (b) $y_{i}$ may now be a primitive value, a value of an array (but not the reference), or a value of a simple object, that is a collection of all values of the fields of a returned object that are in $I_{E}$.

2. Item 1) of Lemma 4 needs to be changed: $\left.t_{i}\right|_{S}$ can now differ from $\left.s_{i}\right|_{S}$, but only on the values in the arrays that $S$ has passed to $E$ (as references to these arrays are not used by $S$ anymore, this will not cause any problem in the results that follow).

With the above changes in the definition of representation (item 1. above) Lemma 5 and Lemma 6 stay true also for $S$ and $E$ like in Theorem 6. Now, these facts can be used to prove Theorem 6 in a very similar way as in the case of Theorem 5.

\section{APPENDIX E. PROOF OF THEOREM 7}

Before we give the proof of Theorem 7, we want to point out some critical points and assumptions that are used in this proof.

1. First, we assume that we have a system CCA2Enc providing the interface $I_{E n c}$ and such that it correctly implements CCA2-secure public encryption scheme (we do not prove correctness of this implementation). We assume that, in particular, the above mentioned implementation does not fail (i.e. always returns the expected result) unless the expected result is too big to fit within an array (recall that the maximum size of an array depends on the security parameter and the function intsize).
We also assume that this encryption scheme is such that the length of a ciphertext is the (polynomially computable) function of the length of the encrypted plaintext and vice versa.

2. It is critical to assume that the Jinja program has unbounded memory, as otherwise the asymptotic notion of security our results are based upon does not make sense.

Now, we shortly present and ideal functionality $\mathscr{F}$ and a real functionality $\mathscr{R}$ for public key encryption in the Turing machine model following [25]

TM functionalities: Different instances of functionalities are distinguished by different ID-s, sent with each request. The functionalities accept the following requests (where the request is written on the input tape of a TM)

1. Initialization-Decryptor: The functionality is supposed to return a public key.

2. Initialization-Encryptor The functionality responds with an comleted message.

3. Encryption $(p k, m)$ : The functionality is supposed to encrypt $m$ with $p k$ and return the result.

4. Decryption $(m)$ : The functionality is supposed to decrypt $m$ using the stored private key.

Both the real and the ideal functionality, on initialization, obtain a corruption bit. Because, in our simulation, the environment never corrupts functionalities, we will skip the description of actions of these functionalities if this bit is set to 1 .

The real functionality $\mathscr{R}$, on initialization (be it Initialization-Decryptor or Initialization-Encryptor), generates a fresh public/private key pair and returns the public key. Then, it uses the private key to decrypt message, and the key $p k$ provided in the encrypt request to encrypt messages.

The ideal functionality, on creation, asks the environment (the simulator) for a crypto algorithm it will use, and creates a key pair using one of the provided algorithms. On encrypt requests, it, similarly to the considered Jinja+ functionality, encrypts an unrelated message of the same length (provided the functionality is not corrupted) and stores it along with the plaintext. On decryption, if the key $p k$ is the same as the public key stored in the functionality, it tries to retrieve the corresponding plaintext from the table (as the IdealPKE does). Otherwise it uses the provided decryption algorithm to decrypt the message.

See [25] for details.

We want to prove that RealPKE realizes IdealPKE w.r.t. $I_{P K E}$. In this proof we will use a result from [25] that $\mathscr{R}$ realizes $\mathscr{F}$. Let $\mathscr{S}$ be the simulator used in the realization proof in [25]. The simulator for IdealPKE we will use in the proof is $S=$ CCA2Enc, as described above.

Let $E$ be a bounded-environment with $I_{P K E} \vdash E$.

Simulating E: We define a Turing machine $M_{E}$ that simulates $E$. Clearly, every complete Jinja+ program can be simulated by Turing machine. Moreover, if a program 
is bounded (for a given intsize), then its simulation is also polynomial (recall that a run with security parameter $\eta$ uses integers of maximal size intsize $(\eta)$; operations on integers of this size can be polynomially simulated by a Turing machine).

In our case, however, the system $E$ we consider is not a complete Jinja+ program; it interacts with another system (such as RealPKE or IdealPKE). Therefore we assume that $M_{E}$ communicates with another Turing machine (or more generally, a system of Turing machines).

The machine $M_{E}$ is defined in such a way that it maintains a representation of a Jinja+ state, the state of $E$. In this representation, references are represented by consecutive identifiers. We distinguish two types of references: those pointing to an internal object, that is instances of a classes defined in $E$ or an arrays, and those pointing to an $e x$ ternal object which can be either instances of Encryptor of Decryptor. For each reference to an internal object, a representation of this object is remembered by $M_{E}$. For references to external objects this is not the case (some additional information, however, can be stored along with these references; see below). A method call for an internal reference is modeled internally in $M_{E}$; a method call to an external object is realized by triggering another Turing machine.

When the simulation of $E$ by $M_{E}$ if finished, this machine outputs the value of the (simulated) variable result.

Method invocations for external references are simulated in the following way:

1. Creating a new instance of Decryptor: $M_{E}$ creates a new instance of Decryptor (Turing machine) by sending the Initialization-Decryptor request with a fresh identifier $i d$. This identifier will be used as the reference to this object. $M_{E}$ waits then for a response with a public key. This key is stored together with $i d$.

2. Decryptor.getEncryptor for an object represented by $i d: M_{E}$ creates a new instance of Encryptor (TM) by sending the Initialization-Encryptor request with id and a fresh identifier $i d^{\prime}$, which will serve as the reference to this object. The identifier $i d^{\prime}$ is stored together with $i d$.

3. Decryptor.decrypt for an object represented by id and array $r: M_{E}$ sends Encryption request to machine $i d$ with the data stored under $r$, and waits for the response. A response is a sequence of bytes. $M_{E}$ simulates creation of a new array and copies the obtained byte-sting to this array.

4. Encryptor.encrypt for an object represented by $i d^{\prime}$ and array $r: M_{E}$ retrieves (the decryptor $i d$ and) the public key associated with $i d^{\prime}$ and uses it in the request Encrypt along with $i d, i d^{\prime}$ and the data stored under $r$. A response is a sequence of bytes. $M_{E}$ simulates creation of a new array and copies the obtained byte-sting to this array.

5. Encryptor.getPublickey for an object represented by $i d^{\prime}: M_{E}$ retrieves the public key associated with the encryptor (without any external call).

Representing runs: Let $T$ be either the system RealPKE or the system $(S$. IdealPKE). Let $u$ be a random input (a sequence of bits) and $\eta$ be a security parameter. Like in Appendix D-A, the (deterministic) finite run $\rho$ of $E \cdot T$ with random input $u$ and security parameter $\eta$ can be represented as

$$
A_{1}\left[s_{1}, x_{1}\right] B_{1}\left[t_{1}, y_{1}\right] A_{2} \cdots B_{n-1}\left[t_{n-1}, y_{n-1}\right] A_{n}\left[s_{n}\right]
$$

where

- Every $A_{i}$ is a part of the run (a sequence of configurations) where only expressions originating from $E$ are reduced, i.e. all the transitions in $A_{i}$ are labelled with names of classes defined in $E$. Every $A_{i}$, except for the last one, ends with a state of the form $\left(e_{i}\left[e_{i}^{\prime}\right], s_{i}\right)$ where the subexpression $e_{i}^{\prime}$ is about to be rewritten by a method invocation rule.

- Every $B_{i}$ is a part of run where only expressions originating from $T$ are reduced. It begins with $\left(e_{i}\left[\left\{e_{i}^{\prime \prime}\right\}_{D}\right], s_{i}\right)$, where $\left\{e_{i}^{\prime \prime}\right\}_{D}$ is the block obtained by applying the method invocation rule to $e_{i}^{\prime}$ for some class $D$ defined in $T$ (it depends only on $\left.e_{i}^{\prime}\right)$, and ends with $\left(e_{i}\left[\left\{v_{i}\right\}_{D}\right], \bar{s}_{i}\right)$, where $v$ is a value (that is return by the method).

- $s_{i}$ and $t_{i}$ are the states after $A_{i}$ and $B_{i}$, respectively.

- By $x_{i}$ we denote the invocation data consisting of the name of the called method and the values passed as arguments (if an argument is of type byte[] then $x_{i}$ contains the values in the array, not the reference to this array). This data is determined by $e_{i}$ and $s_{i}$.

- By $y_{i}$ we denote the return value (again, if an array is returned, then $y_{i}$ contains the values in this array, not the reference). This return value is determined by $v_{i}$ and $t_{i}$.

Similarly, we represent the (deterministic) execution $\tilde{\rho}$ of the system of Turing machines $M_{E} \mid M_{T}$ with random input $u$ and security parameter $\eta$, where $M_{E}$ is defined above and $M_{T}$ is either $\mathscr{R}$ or $(\mathscr{S} \mid \mathscr{F})$ as

$$
\tilde{A}_{1}\left[\tilde{s}_{1}, \tilde{x}_{1}\right] \tilde{B}_{1}\left[\tilde{t}_{1}, \tilde{y}_{1}\right] \tilde{A}_{2} \cdots \tilde{B}_{n-1}\left[\tilde{t}_{n-1}, \tilde{y}_{n-1}\right] \tilde{A}_{n}\left[s_{n}\right]
$$

where

- Every $\tilde{A}_{i}$ is a part of the run of the system where $M_{E}$ is active. Every $\tilde{A}_{i}$, except for the last one, ends with $M_{E}$ sending data $\tilde{x}_{i}$ to $M_{T}$ (and activating $M_{T}$ ).

- Every $\tilde{B}_{i}$ is a part of the run of the system where $M_{T}$ is active. It ends with $M_{T}$ sending a response $\tilde{y}_{i}$ back to $M_{E}$.

- $\tilde{s}_{i}$ is the state of $M_{E}$ after $\tilde{A}_{i}$ (notice the difference to $s_{i}$ which was the state of the whole system after $A_{i}$ ).

- $\tilde{t}_{i}$ is the state of $M_{T}$ after $\tilde{B}_{i}$ (notice, as above, the difference to $t_{i}$ ).

Let $s=(h, l)$ be a Jinja+ state that occurs in the run $\rho$ of $E \cdot T$. We want to define the part of the state $s$ that "belongs" to $E$ and the part that "belongs" to $T$.

We define $\left.h\right|_{E}$ to be the restriction of $h$ to only those references that, in the run $\rho$, have been created by $E$ or 
have been obtained by $E$ as a return value from a call to $T$. By $\left.h\right|_{T}$ we denote the restriction of $s$ to the remaining references, that is the references in the run $\rho$ that have been created by $T$ but not returned to $E$.

We define $\left.l\right|_{E}$ to be the restriction of $l$ to those (static) variables that are accessible from $E$. Similarly, $\left.l\right|_{T}$ denotes the restriction of $l$ to those static variables that are accessible from $T$. Note that these restrictions are disjoint except for the read-only security parameter ( $T$ does not access any static fields of $E ; E$ does not access any static fields of $T$ ).

We take $\left.s\right|_{E}=\left(\left.h\right|_{E},\left.l\right|_{E}\right)$ and $\left.s\right|_{T}=\left(\left.h\right|_{T},\left.l\right|_{T}\right)$.

Let $\tilde{s}$ be a Jinja state as represented by $M_{E}$ and $s$ be a (real) Jinja state. We say that $\tilde{s}$ represents $s=(h, l)$, written $\tilde{s}=s$, if there is a function $f$ from identifiers (that represent references in $M_{E}$ ) to (Jinja) references (addresses) such that

- the domain of $h$ is $f(X)$ where $X$ is the set of identifiers used by $M_{E}$ to represent references,

- if $\tilde{r} \in X$, then the representation of the object pointed by $\tilde{r}$ agrees with the object pointed by $r$ (in the Jinja state) in the following sense: (i) corresponding fields (in the TM representation and in the Jinja object) of primitive types have the same values, (ii) if a filed of the TM representation contains an identifier $i d$, then the corresponding field of the Jinja object contains $f(i d)$.

- The values of variables in $l$ are-up to mapping $f$ - the same as the values in the TM representation of $l$.

We say that $\tilde{x}_{i}$ matches $x_{i}$, where $\tilde{x}_{i}$ and $x_{i}$ are as above, if the requests $\tilde{x}_{i}$ is the translation of the method invocation $x_{i}$, as specified in the simulation process above. In a similar way, we can say that a response $\tilde{y}_{i}$ matches $y_{i}$.

Relation between Jinja runs and TM runs: Now we are ready to relate the runs of the corresponding Jinja programs and Turing machine systems, as introduced above.

Lemma 7. For every random input $u$ and every security parameter $\eta$ (and $A_{i}, \tilde{A}_{i}, \ldots$ as above) we have:

(a) $\left.s_{i}\right|_{E}=\left.t_{i}\right|_{E}$ and $\left.t_{i}\right|_{T}=\left.s_{i+1}\right|_{T}$,

(b) $\tilde{x}_{i}$ matches $x_{i}$,

(c) $\tilde{y}_{i}$ matches $y_{i}$,

(d) $\left.\tilde{s}_{i} \models s_{i}\right|_{E}$,

(e) $\left.\tilde{t}_{i} \models t_{i}\right|_{T}$,

Item (a) states that sub-states of $E$ and $T$ are separated (the execution of $A_{i}$ does not changes what $T$ can access and the execution of $B_{i}$ does not change what $E$ can access).

Items (b) and (c) state that the components $E$ and $T$ in the Jinja run and the corresponding components in the TM system exchange exactly the same data, up to the provided translation.

Item (d) states that $M_{E}$ correctly simulates $E$ (which is given by the definition of $M_{E}$ ).

Item (e) states that the Jinja program $T$ is functionally equivalent to the corresponding Turing machine $M_{T}$. In particular, for the same input, $\mathscr{R}$ produces the same data as
RealPKE and $\mathscr{S} \mid \mathscr{F}$ produces the same data as $S \cdot$ IdealPKE. This is given by the definition of these systems.

In the reasoning below, we leverage the fact that, without loss of generality, we can assume that $E$, when connected with $T$, never makes requests to $T$ that fail (i.e. never makes method calls that return null). This is because $E$ can compute the expected size of the output message (recall that we assumed that the length of a plaintext and a corresponding ciphertext are polynomially related). Therefore $E$ can predict potential failure and avoid requests that would fail ( $E$ does not lose any information by not executing these requests, as it knows the result up front).

Now, we can observe that a direct consequence of the above lemma (more precisely, of the fact that $\tilde{s}_{n}\left|=s_{n}\right|_{E}$ ) is that the final value of variable result in $\rho$ and $\tilde{\rho}$ is the same and, therefore, these (finite) runs output the same result. As it holds for all random input $u$ and all security parameters $\eta$, up to some negligible function, the system $E \cdot$ RealPKE outputs true with the same probability the system $M_{E} \mid \mathscr{R}$ outputs 1 and the system $E \cdot S \cdot$ IdealPKE outputs true with exactly the same probability the system $M_{E}|\mathscr{S}| \mathscr{F}$ outputs 1. Now, as we know that $M_{E}\left|\mathscr{R} \equiv M_{E}\right| \mathscr{S} \mid \mathscr{F}$, it follows that the probability that true is output by $E$. ReaIPKE and by $E \cdot S \cdot$ IdealPKE is the same up to some negligible value.

\section{APPENDIX F. VERIFICATION USING JOANA}

Program slicing is a well known static analysis technique that is used to capture dependencies between program statements. It can be applied to check a program for sequential termination-insensitive non-interference. However, when the program changes, the analysis has to be redone in order to guarantee non-interference. In this subsection we sketch a proof that under certain restrictions small changes to the program do not change the analysis result with regard to information flow and non-interference. The technique proposed here allows Joana to verify families of programs considered in Section VII.

\section{A. Introduction to Information Flow Control with Slicing}

The concept of program slicing was first introduced by Weiser [40], where he argued about how a programmer tries to determine the relevant program parts responsible for a specific error: He identifies the program statement $s$ where the error occurred and subsequently infers all statements that may have influenced the execution of $s$. These statements are called the backward slice of $s$. Later, Ottenstein and Ottenstein [34] proposed using program dependence graphs (PDG) in order to compute program slices. PDGs are a graph based representation of the program semantics, where a node corresponds to a statement and edges correspond to dependencies between statements.

The Joana project [20], [19] applies a very precise slicer to information flow control for Java programs. It has been 
developed and improved for several years and can analyze medium sized programs up to 80.000 LoC. Joana takes a set of secret (high) input statements and a set of untrusted (low) output statements as arguments and checks if an illegal flow from high to low is possible. Therefore it computes the PDG representation from the program source and marks the PDG nodes that correspond to high or low statements with matching security labels. Then a dataflow analysis ${ }^{2}$ on the PDG propagates the input security labels along the graph edges. Finally Joana checks if any high security label has reached a statement labeled as low output. If no information leaking low statements are found, the program is guaranteed to be secure.

In case of a security violation slicing is used to determine the high input statements involved: All high input statements included in the backward slice of an information leaking low statement are potentially leaked. So whenever information flows from a high statement $h$ to a low statement $l, h$ must be included in the backward slice of $l$. If $l$ is not reachable from $h$ then the absence of information flow is guaranteed.

\section{B. PDG Structure and Computation}

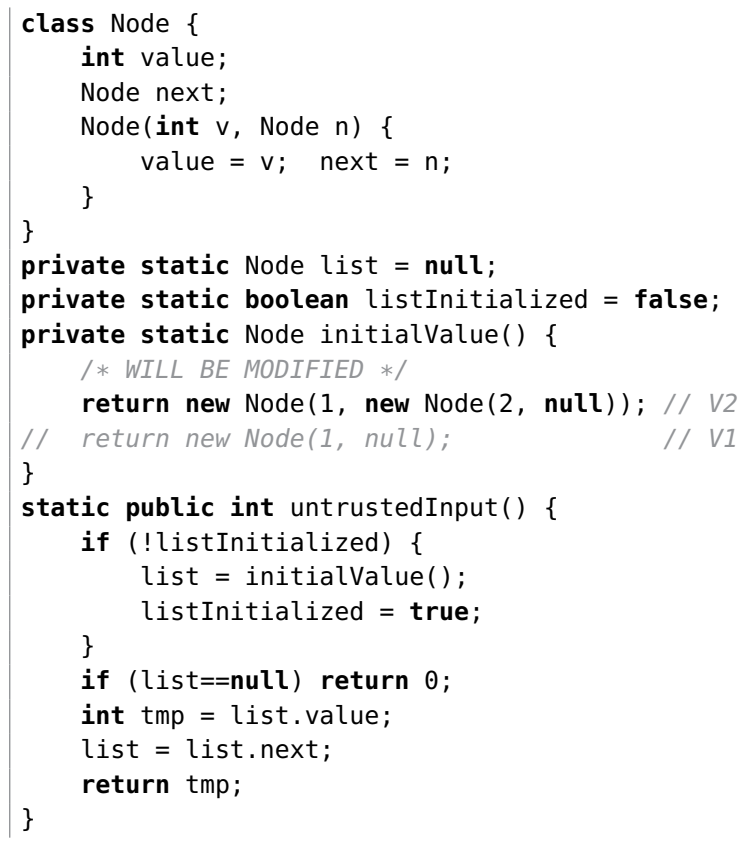

Figure 11. Code that models unknown input in form of a linked list as proposed in section VII.

Figure 13 shows a PDG for the example program in Figure 11 and 12. It contains a node for each statement and additional entry nodes (bold outline) that correspond to method entries. Entry nodes have special fields $I N$ and $O U T$

\footnotetext{
${ }^{2}$ As Joana supports declassification and any kind of security labels that form a lattice, a simple reachability analysis is not enough in general.
}

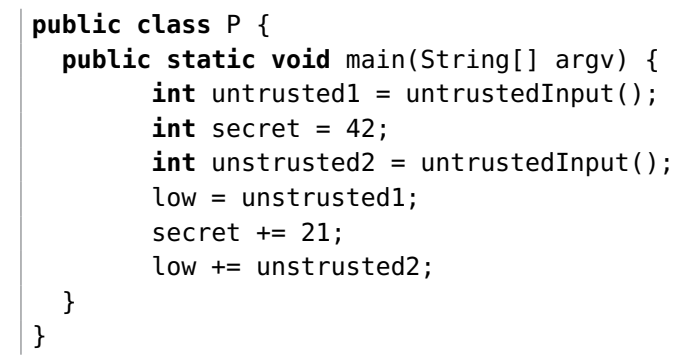

Figure 12. An example of a program without illegal information flow using untrusted input from Figure 11.

representing the input and output of the method. Nodes of method call statements also include those fields representing the values sent to and received from the called method. The nodes of each single method form a subgraph that is only connected to other methods through edges between calls and entry nodes. We distinguish four kinds of edges through which information flow may occur:

- control dependence edge: Statement $n$ is control dependent on $m$ iff the outcome of the execution of $m$ decides if $n$ is executed. E.g. all statements in the body of an ifclause are control dependent on the condition statement. Control dependencies are computed for each method through an intraprocedural analysis on its control flow graph. They capture implicit flow of information.

- data dependence edge: Statement $n$ is data dependent on statement $m$ iff $n$ reads a value that $m$ has produced. This includes the use and definition of variables as well as referenced and modified values on the heap. Data dependencies on heap values are computed with the help of a points-to analysis. A points-to analysis computes for each heap reference at which locations on the heap it may point to. When statement $m$ modifies the same location statement $n$ reads and $n$ is executed after $m, n$ is data dependent on $m$. Data dependencies connect only statements that belong to the same method. In order to capture flow between methods, call and parameter edges are used.

- call edge: A call edge connects a method call statement to the entry node of the called method.

- parameter and heap dependence edge: Parameter and heap dependence edges capture the flow of information from a method call to the called method and back. They include the parameters passed into the method and the return value passed back to the callsite and also heap locations referenced and modified by the method.

\section{Problem: Parameterized initialization}

We consider programs $P_{\vec{u}}$ consisting two parts: $P_{\text {main }}$ and $E_{\vec{u}}$, where $P_{\text {main }}$ is a system that meets the conditions given below and $E_{\vec{u}}$ is as in Figure 11, for the variant of the method initialValue $\vec{u}_{0}$ determined by a sequence $\vec{u}$, 
as in Section VII. We require (which is consistent with assumption of Section VII) that

- $P_{\text {main }}$ does not reference nor modify the variable list,

- $P_{\text {main }}$ does not create object of class Node,

Note that with these conditions the following statements are true:

- Only untrustedInput references and modifies list.

- A single static method initialValue $\vec{u}_{0}$ creates all list elements and initializes the variable list.

- The constructor for elements of list is only called inside initialValue $\overrightarrow{\vec{u}}_{0}$.

- initialValue $\vec{u}_{0}$ has no parameters and does not reference or modify any static variable.

- neither untrustedInput nor initialValue $\vec{u}_{0}$ contain statements that reference high (secret) or low variables.

Using these properties of programs $P$ as described above, we can prove the following result.

Proposition 1. For all $P_{\text {main }}$ such that $P_{\vec{u}}=P_{\text {main }} \cdot E_{\vec{u}}$ comply with the requirements stated above, if $P_{\vec{u}_{0}}$ does not contain illegal information flow, for some sequence $\vec{u}_{0}$, then $P_{\vec{u}}$ does not contain illegal information flow for all $\vec{u}$.

Proposition 1 enables us to reason about a family of programs by analysing only a singe instance of it. We take some specification of $\vec{u}_{0}$ and analyze $P_{\vec{u}_{0}}$ If the analysis guarantees security in this case, we know due to Proposition 1 that the program is secure for all versions of initialValue $\vec{u}_{0}$ fulfilling the restrictions.

Because programs $T_{\vec{u}}$ we consider in Section IX-B comply with the assumptions of the above theorem, we can show non-interference of this family of programs by showing noninterference of only one specific instance. Indeed, we have used Joana to verify that such an instance (and hence the whole family of programs) is indeed secure.

\section{Example}

We can take the program from Figure 12 as $P_{\text {main }}$. Indeed all the above requirements are met for $P_{\vec{u}}=P_{\text {main }} \cdot E_{\vec{u}}$.

This program includes reading and writing of untrusted values as well as a computation on a secret value. Figure 13 contains the corresponding PDG for $\vec{u}=1,2$. This program is considered secure, because the PDG contains no connection from the secret value in line 28 to a statement that leaks to untrusted output (line 30 and 32).

\section{E. Proof sketch of Proposition 1}

Let us assume that the program $P_{\vec{u}_{0}}=P_{\text {main }} \cdot E_{\vec{u}_{0}}$, for some $\vec{u}_{0}$ is secure. Note that we have assumed a set of statements $S_{\text {secret }} \subseteq P_{\text {main }}$ that refers to secret values in $P_{\vec{u}_{0}}$ and a set of statements $S_{\text {out }} \subseteq P_{\text {main }}$ that refer to untrusted output (e.g. changing the value of a low variable). As $P_{\vec{u}_{0}}$ is guaranteed to be secure, we also know that there is no path

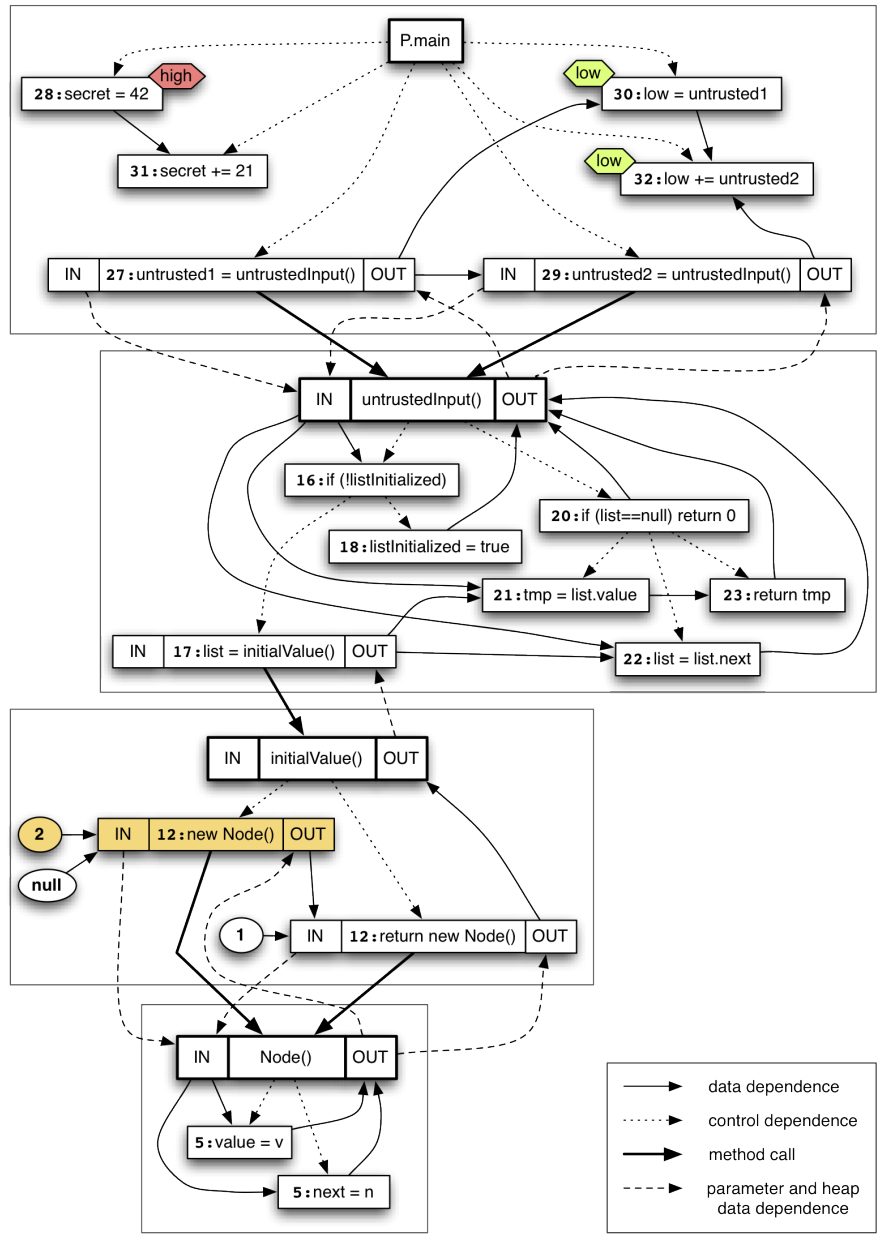

Figure 13. A PDG of the example program from Figure 11 and 12. The colored nodes are the additional nodes that occur if version $V 2$ of initialValue $\vec{u}_{0}$ is chosen instead of $V 1$.

in the corresponding $P D G_{P_{\vec{u}_{0}}}$ from secret values to untrusted output:

$$
\forall l \in S_{\text {out }}: \text { slice }_{P D G_{P_{\vec{u}_{0}}}}(l) \cap S_{\text {secret }}=\{\}
$$

If we use $\operatorname{Path}\left(P D G_{P_{\vec{u}_{0}}}\right)$ as the set of all possible paths in $P D G_{P_{\vec{u}_{0}}}$ we get:

$\forall h \in S_{\text {secret }} \forall l \in S_{\text {out }} \forall p \in \operatorname{Path}\left(\operatorname{PDG}_{P_{\vec{u}_{0}}}\right): h \in p \Longrightarrow l \notin p_{\mid h}$

Let

$p_{\mid h}=\left(p_{1} \rightarrow \ldots \rightarrow h \rightarrow p_{i} \rightarrow \ldots \rightarrow p_{n}\right)_{\mid h}=\left(h \rightarrow p_{i} \rightarrow \ldots \rightarrow p_{n}\right)$

We will use the following, auxiliary lemma:

Lemma 8. Changes in the PDG local to initialValue $\vec{u}_{0}$ are not affecting the security guarantee.

Proof: If the PDG local to initialValue $\vec{u}_{0}$ has changed, either previously existing nodes or edges were removed or new ones were introduced. Removing nodes or edges has no effect on the security guarantee as it 
only reduces the number of possible paths in the whole PDG. So no additional flow can be introduced. Additional nodes or edges inside the PDG part of initialValue $\vec{u}_{0}$ does increase the number of possible paths inside the method, but not the number of paths leading to or leaving from it. Because initialValue $\vec{u}_{0} \cap S_{\text {secret }}=\{\}$ and initialValue $\vec{u}_{0} \cap S_{\text {out }}=\{\}$ we get that no illegal flow can not start or end inside of initialValue $\vec{u}_{0}$. Due to the restrictions we know that initialValue $\vec{u}_{0}$ does not take any parameters as arguments and that it does not reference any static variables. So no parameter and heap dependence edges are connected to the entry node of initialValue $\vec{u}_{0}$. The only possible paths from a secret source to an untrusted output through initialValue $\vec{u}_{0}$ have to enter through the call edge and to leave through the parameter and heap dependence of the OUT field. Because any output of a method is dependent on the method execution, there is always a path from the entry node to its $O U T$ field. So additional nodes or edges inside initialValue $\vec{u}_{0}$ do not introduce new flow from $S_{\text {secret }}$ to $S_{\text {out }}$.

Now we change initialValue $\vec{u}_{0}$ of $P_{\vec{u}_{0}}$ to initialValue $_{\vec{u}}$, given by some $\vec{u}$, and assume that the resulting program $P_{\vec{u}}=P_{\text {main }} \cup E_{\vec{u}}$ contains an illegal flow. Then we show by contradiction that this is not possible and thus $P_{\vec{u}}$ must be secure.

By the above assumption

$$
\exists h \in S_{\text {secret }} \exists l \in S_{\text {out }}: h \in \text { slice }_{P D G_{P_{\vec{u}}}}(l)
$$

which means there must be a path $p$ from $h$ to $l$ in the changed PDG that was not part of the original.

$$
\exists p \in \operatorname{Path}\left(P D G_{P_{\vec{u}}}\right): h \in p \wedge l \in p_{\mid h} \wedge p \notin \operatorname{Path}\left(P D G_{P_{\vec{u}_{0}}}\right)
$$

Path $p$ is a list of instructions that are connected through dependencies. We are now going to show that any of these dependencies must already have been part of $P D G_{P_{\vec{u}_{0}}}$.

If $p \notin \operatorname{Path}\left(P D G_{P_{\vec{u}_{0}}}\right)$ then $p$ must contain at least one edge $n_{1} \rightarrow n_{2}$ that is not in $P D G_{P_{\vec{u}_{0}}}$.

$$
p \notin \operatorname{Path}\left(P D G_{P_{\vec{u}_{0}}}\right) \Longrightarrow \exists n_{1} \rightarrow n_{2} \in p \mid n_{1} \rightarrow n_{2} \notin P D G_{P_{\vec{u}_{0}}}
$$

Now we consider four different cases.

Case $n_{1} \notin P D G_{P_{\vec{u}_{0}}}, n_{2} \notin P D G_{P_{\vec{u}_{0}}}$. Both nodes have not been part of $P D G_{P_{\vec{u}_{0}}}$. Because initialValue $\vec{u}_{\vec{u}}$ is the only changed part of $P_{\vec{u}}$ we know that $n_{1}, n_{2} \in$ initialValue $\vec{u}_{\vec{u}}$. Due to Lemma 8 this edge can not introduce new illegal flow.
Case $n_{1} \notin P D G_{P_{\vec{u}_{0}}}, n_{2} \in P D G_{P_{\vec{u}_{0}}}$. Because initialValue $\vec{u}_{\vec{u}}$ is the only changed part of $P_{\vec{u}}$ we know that $n_{1} \in$ initialValue $_{\vec{u}}$. So due to Lemma 8 this edge cannot introduce new illegal flow.

Case $n_{1} \in P D G_{P_{\vec{u}_{0}}}, n_{2} \notin P D G_{P_{\vec{u}_{0}}} \cdot n_{2} \in$ initialValue $\vec{u}_{\vec{u}}$ so no new illegal flow is introduced with this edge.

Case $n_{1}, n_{2} \in P D G_{P_{\vec{u}_{0}}}$. Only the edge between $n_{1}$ and $n_{2}$ is new in $P D G_{P_{\vec{u}}}$. Because of Lemma 8 we only have to consider the case $n_{1} \rightarrow n_{2} \notin$ initialValue $\vec{u}$. The new edge may be of four different kinds:

- control dependence edge: Control dependencies are computed per method using the control flow graph. No method in $P_{\vec{u}_{0}} \backslash$ initialValue $\vec{u}_{0}$ (that is no method of $P_{\vec{u}_{0}}$ except for initialValue $\vec{u}_{0}$ ) has been changed, so the control flow is unchanged and thus no control dependencies have changed. So $n_{1} \rightarrow n_{2}$ cannot be a control dependency.

- data dependence edge: Data dependencies are either caused through use and definition of variables or references and modification to heap locations. $P_{\vec{u}} \backslash$ initialValue $\vec{u}_{\vec{u}}$ contains no new statements and thus no additional uses and definitions of variables. So the additional data dependence cannot stem from them and has to be introduced through accesses to heap locations. The only heap locations that have changed are the elements of list. Our restrictions forbid references to this list in $P_{\text {main }}$, so $n_{1} \rightarrow n_{2}$ cannot be a data dependency.

- call edge: $P_{\vec{u}} \backslash$ initialValue $\vec{u}$ contains no new statements and thus no new call statements. So $n_{1} \rightarrow n_{2}$ cannot be a call dependency.

- parameter and heap dependence edge: These edges are caused by passing parameters into methods and returning method results. As $P_{\vec{u}} \backslash$ initialValue $\vec{u}$ is not changed, no method signatures and calls are altered. So $n_{1} \rightarrow n_{2}$ cannot be caused by additionally passed parameter or returned results. The last remaining possibility is that the edge is caused by a change in the referenced or modified heap locations. Due to the restrictions we know that only heap locations referring to elements of list may have changed. As $P_{\text {main }}$ does not contain references to list or its elements, $n_{1} \rightarrow n_{2}$ is no parameter or heap dependency.

We showed that $n_{1} \rightarrow n_{2}$ cannot exists, thus path $p$ cannot exist and so no illegal information flow can be introduced by changes in initialValue $\vec{u}_{0}$. 